\title{
Holographic Sensors in Biotechnology
}

\author{
Sam Davies, Yubing Hu, * Nan Jiang, * Jeff Blyth, Monika Kaminska, Yunzheng Liu, \\ and Ali K. Yetisen
}

As populations expand worldwide, medical care will need to diversify its data collection techniques to be able to provide adequate healthcare to global populations, this can be achieved through point-of-care analysis by wearable analytical devices. Holographic sensors are reusable optical biosensors with the capability to continuously monitor variations, generating the prospect of in vivo monitoring of patient homeostasis. Holographic optical sensors have emerged as an opportunity for low cost and real-time point-of-care analysis of biomarkers to be realized. This review aims to summarize the fundamentals and fabrications of holographic sensors; a key focus will be directed to examining the biotechnology applications in a variety of analytical settings. Techniques covered include surface relief gratings, inverse opals, metal nanoparticle and nanoparticle free holographic sensors. This article provides an overview of holographic biosensing in applications such as $\mathrm{pH}$, alcohol, ion, glucose, and drug detection, alongside antibiotic monitoring. Details of developments in fabrication and sensitizing techniques will be examined and how they have improved the applicability of holographic sensors to point-of-care analytics. Although holographic sensors have made significant progress in recent years, the current challenges, and requirements for advanced holographic technology to fulfil their future potential applications in biomedical devices will be discussed.

and biosensing to mention a few. ${ }^{[1-7]}$ Currently, a rise in value of the optical sensor market can be observed, with further increase being projected. The global optical sensors market is expected to grow at $15 \%$ of Compound Annual Growth Rate (CAGR) between 2016 and 2022 to reach USD 20 Billion by $2022 .{ }^{[8]}$ Some of the factors on this growth can be attributed to: rise in wireless sensing technologies, need for enhanced security and safety solutions, and better alternatives for conventional sensing technologies. ${ }^{[9]}$ The benefits of holographic sensors which give them a market advantage include high accuracy and reliability, immunity to electromagnetic interference (EMI), and project high return on investment in the long run. ${ }^{[10]}$ Moreover, holographic devices are usually low cost, easily transportable, and offer less complex readouts than conventional sensing methods. ${ }^{[8]}$ They also require less energy input, and therefore are more environmentally friendly. ${ }^{[9]}$ However, lack of awareness, high initial investment, and

\section{Introduction}

Holographic sensors belong to the category of optical sensors, which utilize light as a method for detailing variation in analytes or conditions. The optical sensing platform is seen to be of increasing in significance in recent years with applications spanning industrial, environmental, food monitoring systems,

S. Davies, Y. Hu, J. Blyth, M. Kaminska, Y. Liu, A. K. Yetisen

Department of Chemical Engineering

Imperial College London

London SW7 2BU, UK

E-mail: yubing.hu@imperial.ac.uk

N. Jiang

West China School of Basic Medical Sciences and Forensic Medicine

Sichuan University

Chengdu 610041, China

E-mail: jiangnansophia@scu.edu.cn

The ORCID identification number(s) for the author(s) of this article can be found under https://doi.org/10.1002/adfm.202105645.

(C) 2021 The Authors. Advanced Functional Materials published by Wiley-VCH GmbH. This is an open access article under the terms of the Creative Commons Attribution License, which permits use, distribution and reproduction in any medium, provided the original work is properly cited.

The copyright line for this article was changed on 23 August 2021 after original online publication.

DOI: 10.1002/adfm.202105645 insufficient technological standards are aspects that can be seen as limitations in market growth of optical sensors and should be further addressed. ${ }^{[9]}$

\subsection{History of Holographic Sensors}

Optical diffractive structures are present in numerous common objects and animals observed in the world around us. Animal and insect species often display structural coloration on their bodies giving them iridescent color (Figure 1a). ${ }^{[11-14]}$ An example of this phenomenon is the wings of Morpho butterflies (Figure 1b). ${ }^{[14]}$ Electron microscopy of the wings of these insects reveals the periodic structure responsible for the coloration (Figure $1 \mathrm{~b}$-ii). ${ }^{[15]}$ The surface of the wings is covered in a highly ordered 3D array of nanostructures which reflect a narrow band interaction with light in the blue region of the spectrum. ${ }^{[15]}$

Holographic recordings have been established since the mid20th century. Dennis Gabor a British-Hungarian scientist often referred to as the "Father of Holography," initially was able to generate holographic gratings using the interference pattern between a reflected wave front and reference wave emitted from a relatively uncoherent light source. ${ }^{[17]}$ It was for this work that Gabor alone was awarded a Nobel Prize in 1971. Although Gabor initially published his findings in 1947, the work as unable to be utilized widely due to the technology at the time 
a) i)

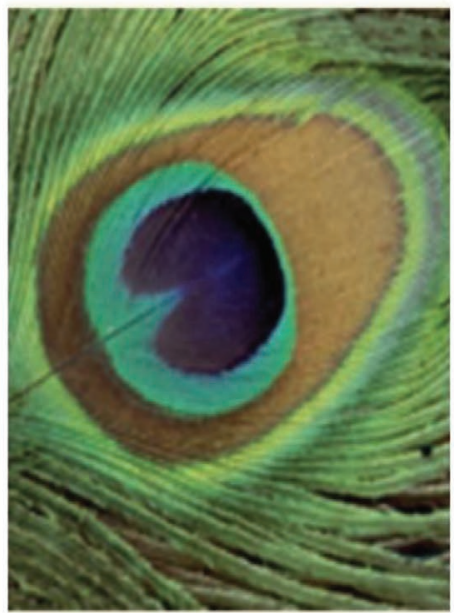

ii)

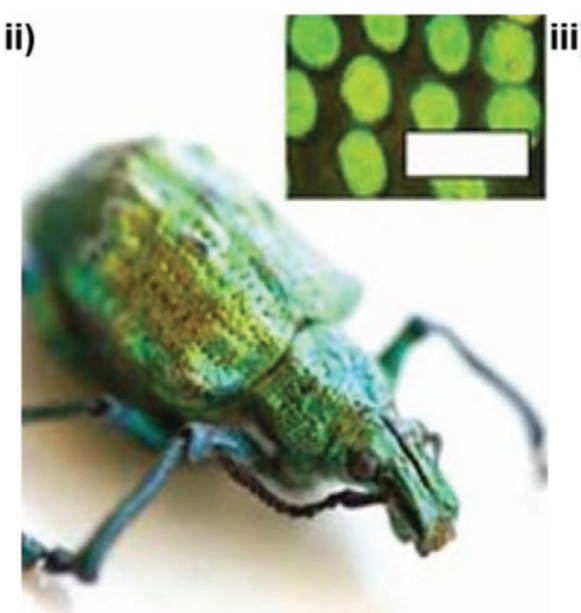

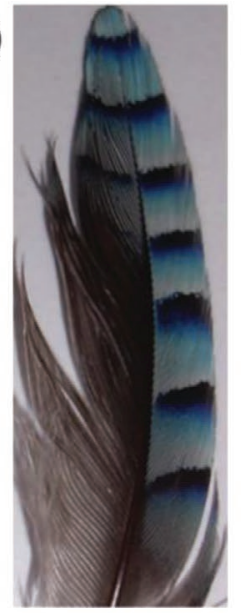

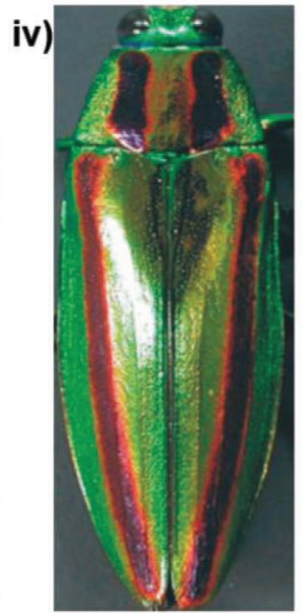

b) i)
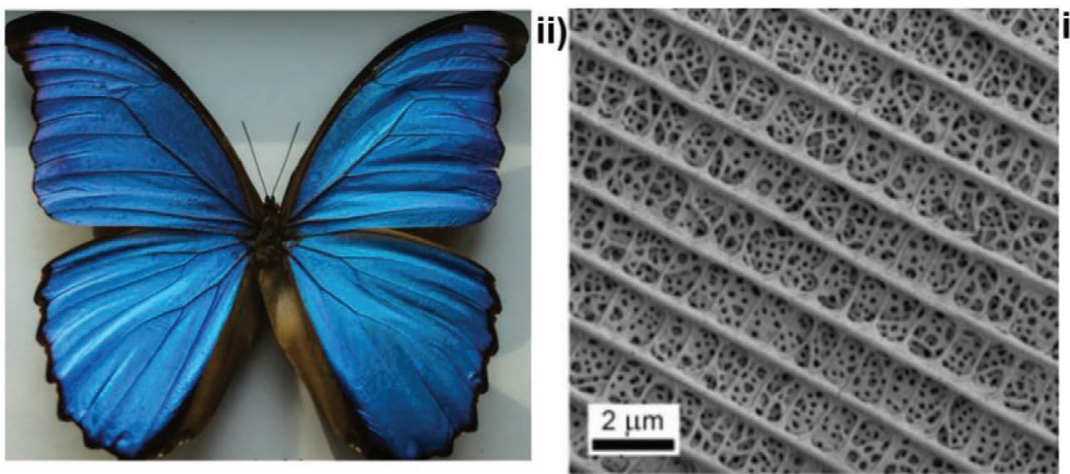

ii)

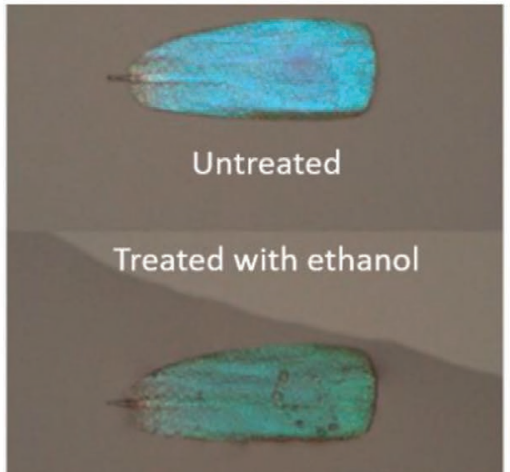

Figure 1. Demonstration of the natural occurrences of optical structures and the responsive nature of the gratings. a) i) Structural colouration observed in the iridescent colour of peacock feathers. Reproduced with permission. ${ }^{[12]}$ Copyright 2012, Springer Nature; ii) Photograph of weevil L. Augustus, inset demonstrates the green coloured photonic scales. Reproduced with permission. [11] Copyright 2011, Wiley-VCH; iii) Periodically patterned Jacy feather. Reproduced with permission. ${ }^{[16]}$ Copyright 2015, Nature Scientific Reports; iv) Photograph of Japanese jewel beetle. Reproduced with permission. ${ }^{[130]}$ Copyright 2011. The Authors, published by The Royal Chemical Society. b) i) Photograph of Morpho butterfly. Reproduced with permission. [131] Copyright 2018, Springer Nature; ii) Transmission electron microscopy (TEM) image of wing scales of a Morpho butterfly, iii) Morpho butterfly wing as observed in the wild (top), Morpho butterfly wing treated with ethanol (bottom). Reproduced under the terms and conditions of the Creative Commons Attribution (CC BY) license. ${ }^{[15]}$ Copyright 2018, The Authors, published by MDPI.

being unable to produce monochromatic coherent light. The explosion of holographic research was later in 1960, with the invention of the laser, ${ }^{[18]}$ which allowed Emmett Leith to record the first 3D hologram. ${ }^{[19]}$ Holographic recording can be considered similar to normal photography where light is directed onto a photosensitive medium which records the incident light which, post development, shows an image of the scene. Normal photography records a single front of light whereas holographic sensors utilize a complex interference pattern between two coherent waves. One wave front is reflected from the object, which contains the image wave, the other light is a reference beam. ${ }^{[20]}$ Both components are directed onto the photosensitive recording material and the interference pattern between the two waves gives rise to the holographic recording containing a 3D image of the object. Up until this point holographic gratings served data storage and artistic purposes, in modern science holographic sensors have found application in developing sensors. By utilizing smart materials as a substrate for simple holographic structures wavelength selective Bragg mirrors with tunable responses to chemical and physical stimuli are achievable. ${ }^{[21]}$ The variation in wavelength can be calibrated against the volumetric responses to physical or chemical stimuli within the hydrogel film. The development of both smart materials such as functionalized hydrogels and holographic sensors have worked in tandem. The functionalization of films to correspond to change in biomarker concentrations allows for simple biosensor to be developed capable of directly interacting with the chemical properties of analytes unlike many other sensing platforms which often rely on changes induced by the desired analyte possibly offering greater opportunity for high selectivity.

\subsection{Requirement for Holographic Sensors in Biomedical Applications}

Recently, a lot of focus has been placed on the practical aspects of sensing, for instance, introducing simple readout methods to improve affordability and accessibility to healthcare worldwide. ${ }^{[22,23]}$ Holographic sensors can deliver label free sensing to remove a number of complex processing steps often required by conventional chemical analysis, therefore reducing the level of training and intricate equipment required to obtain reliable data. ${ }^{[24]}$ Smartphone applications can be designed to convert sensor response into a qualitative result. ${ }^{[25]}$ This in tandem with 
the opportunity to fabricate sensors using biocompatible fibers. Biocompatibility can allow for the long-term exposure of biological systems to sensing platforms, offering truly continuous monitoring. These characteristics offer the opportunity for holographic sensors to be truly disruptive in the point-of-care biosensor industry.

There has been rapid development in the use of functionalized nanostructured surfaces to allow for quantitative and accurate measurement of biological analytes with the development of sophisticated substrates such as hydrogel based ionic skins. ${ }^{[26]}$ Holographic sensors for analyzing hydration, ${ }^{[17]}$ monitoring respiration, ${ }^{[18]}$ lactate, ${ }^{[27]}$ urinalysis, ${ }^{[28]}$ tear fluid analysis, ${ }^{[29,30]}$ and alcohol intoxication ${ }^{[31]}$ within the body has been highly investigated in recent years due to the growing drive to personalized medical care. ${ }^{[32]}$ The development of these devices offers the possibility for healthcare providers to analyze the realtime concentrations of biomarkers using simple inexpensive analytical equipment. This can pair with advances in understanding how genetic composition affects our susceptibility to ailments, we are moving towards an era where treatments will be more tailored to the patient than ever previously possible. ${ }^{[33]}$ Due to the inherent holographic sensing mechanism utilizing the reversible binding of analytes continuous monitoring is achievable, giving a whole new dynamic to the field of medical treatment. Offering real-time data of a patient's condition, permits the personalization of medical treatment. An example would be in the absorption, distribution, elimination, and metabolism of drugs, the rates of these processes vary in every individual. If a dose regime has a narrow therapeutic index, this could be due to high interindividual drug variability or due to toxicity risks, dosing accuracy is vital. ${ }^{[34,35]}$ Conventional chemical analysis methods for example highperformance liquid chromatography and liquid chromatography-mass spectroscopy would not be viable due to cost and training requirements. Holographic sensors are classed as an optical sensor, utilizing light as a medium to indicate variation of analytes. Holographic sensors are responsive to biological and chemical stimuli generating a simple, low cost, time effective sensing platform, the widespread deployment to patients could report variation in metabolites directly to patients or medical professionals remotely. This capacity for a patient or doctor to monitor the body more frequently than in conventional appointments allows treatment to be administered when required rather than utilizing a general time frame, ensuring the maintenance of the therapeutic effect. ${ }^{[36,37]}$ In the previous two years we have seen how powerful point-of-care analytics have been in the COVID-19 pandemic. ${ }^{[38]}$ Rapid lateral flow devices have allowed global populations to be assured of their infection status without time consuming polymerase chain reaction tests, this benefit could be applied to several illnesses through the application of holographic devices.

Holographic sensors exhibit characteristics specifically beneficial to sensing functions when compared to their electrochemical counterparts. The lack of requirement for electrical currents or large metallic components of holographic sensors, permits numerous applications within demanding environments, that is, high magnetic fields. ${ }^{[39-41]}$ In modern medicine, Magnetic Resonance Imaging (MRI) scans have become a powerful diagnostic tool dependent on the use of high magnetic fields. ${ }^{[42]}$ The strong magnetic field by which MRI scan images are generated can lead to several complications when metallic components of electrochemical cells are introduced. Metals interacting with the magnetic field generates distortions known as "artefacts" within the images obtained, giving unreliable readouts which can lead to misdiagnosis. In tandem with this interference, metals within a magnetic field will be physically pulled towards the magnet source, alongside electromagnetic heating to temperatures capable of causing permanent damage to the patient. ${ }^{[43]}$ Optical sensors are able to remove this interference and risk due to the absence of metallic wires and the use of only light as the sensing medium. In future, this will allow for new avenues of diagnosis to be explored, for example analysis of biological processes within the brain whilst simultaneously recording MRI scans. This development creates a possibility of obtaining previously inaccessible data about degenerative diseases such as Alzheimer's and dementia furthering our understanding. ${ }^{[44]}$

\subsection{Optical Properties and Sensitization}

The fabrication methods explored in this review contain periodic nanostructures with varying refractive index capable of interacting with light to generate an optical response. Change in refractive index (RI) alters light passing through layered structures giving rise to structural coloration as a Bragg mirror. ${ }^{[45]}$ Refractive index is defined by Equation (1) where $n$ is the refractive index, $c$ is the speed of light and $v$ is the phase velocity of light.

$n=\frac{c}{v}$

The color observed is dependent on the fringe spacing between changes in refractive index of these scales, defined by Bragg law (Equation 2). This interaction occurs when light interacts with a periodic structure, causing a specific wavelength of light to be scattered by the matrix. Alteration of this $d$ spacing changes the wavelength of light reflected (Figure 2a). This correlates with the responses seen from the other optical structures whereby their surroundings can alter the response observed.

$n \lambda=2 d \sin (\theta)$

The recording of holographic sensors is dependent upon the use of constructive interference between incident and reflected light to generate points of high and low intensity (Figure 2b). ${ }^{[46]}$ A range of laser wavelengths can be used to generate the holographic grating. The interference between the two propagating waves to generates nodes and anti-nodes where the fringe structures are recorded. ${ }^{[46]}$ This dependency of initial fringe spacing on the recording laser has a direct impact on the replay wavelength observed. Holography has origins in the chemistry utilized in photography, but significant differences clearly separate the two processes in their application and future scope. Photographic images are the recording of a wave front reflecting from an object, whereas holographic sensors depend upon the recording of the interference pattern of the beam reflected from the object and a reference beam. The interference between these two counter-propagating beams gives rise to points of high and low intensity which creates a perspective in the image recorded. By controlling the way in which these two beams interfere holographic gratings can be recorded in two different 


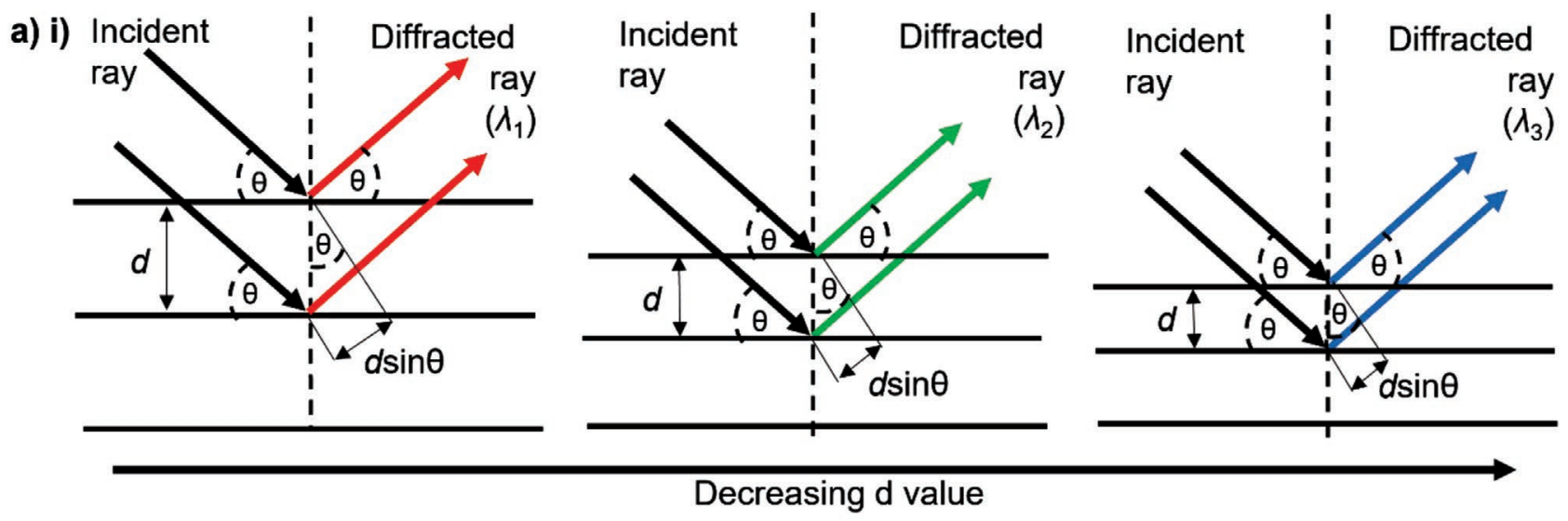

b)

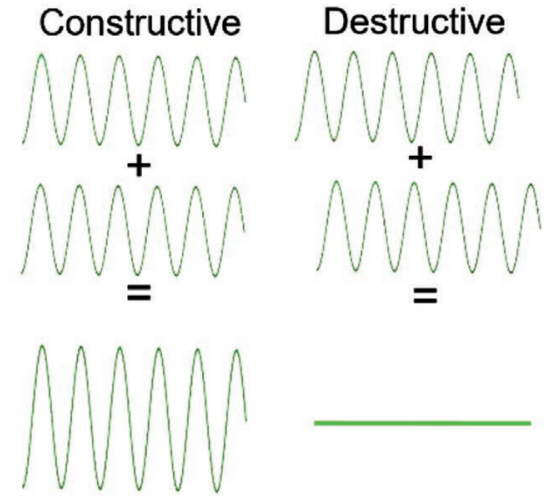

c) i)

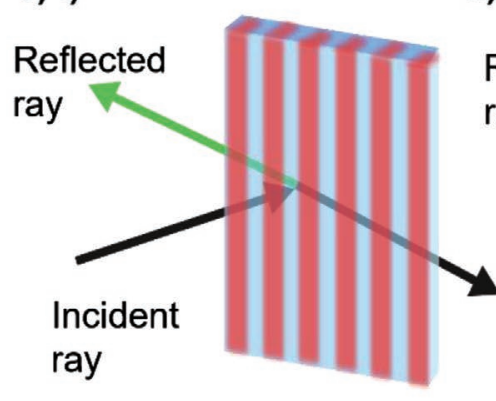

ii)

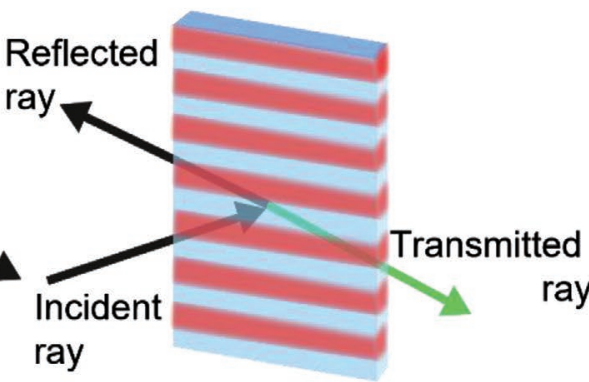

Figure 2. Overview of some of the fundamentals of holographic interactions with light. a) Dynamic relationship of reducing layer spacing and the tuning of the diffracted wavelength ( $\lambda$ ); b) Constructive and destructive interference between wavelengths; c) Diffraction gratings recorded in i) reflection and ii) transmission and how the grating orientation affects the interaction with light.

conformations, either reflection or transmission. The main difference between these two recording methods is the direction from which the two incident beams interact. If the two beams come from opposing sides, then the hologram recorded will be in reflection, whereas if the two beams come from the same side the hologram will be in transmission. The resultant interference patterns between these two arrangements give rise to the gratings illustrated in Figure 2c. These two conformations differ in how they interact with light, reflection gratins will selectively reflect a single wavelength dependent upon the spacing of the fringes, whereas transmission gratings will create a diffraction pattern spreading the light that passes through them dependent upon the $d$ spacing. ${ }^{466]}$

Sensitization to specific biomarkers is achieved by utilizing responsive hydrogel materials which initiate a volumetric change upon the introduction of external stimulus. ${ }^{[4]}$ These polymer networks are modified with sensitizing moieties, receptive to specific analytes, which stimulate swelling of a material dependent on the concentration of an analyte in the test solution. The volumetric response of the network leads to the alteration of layer spacing and therefore a variation in the optical properties observed. This review will aim to look at the various methods of formulating nanostructures within smart hydrogel networks and how different formulation methodologies can be utilized to generate a variety of sensing platforms.

The holographic sensing mechanism is intrinsically built into the structure, not requiring an external electrical input to stimulate detection offering a route to continuous monitoring of analytes increasing the level of collectable data. Holographic colorimetric sensing permits users to observe variation of specific analytes via the reflected wavelength variation without the requirement for expensive analytical equipment. Alongside this, outside the biosensing field, these properties can allow for a rapid identification of contaminates or leaks in industrial settings without human stimulus, which may both save lives and highlight the presence of an issue prior to a major incident. ${ }^{[48,49]}$ With electrochemical cells, it is a necessity to recalibrate the device regularly due to signal drift. ${ }^{50]}$ Recalibration although tedious, is less of an issue for nonmedical devices or monitoring of samples, but if a device is to be implanted recalibration becomes a significantly more difficult task. Optical structures rarely require a re-calibration once formulated as they are less prone to signal drift. ${ }^{[50]}$ Reduction in the requirement for recalibration decreases overall impact on patients day to day activities. Electrochemical cells are currently considered to be capable of a higher level of accuracy in the of monitoring analytes than previous holographic structures. This review aims to explore the current developments of holographic sensors and how they are challenging the current technologies in the field.

\section{Fabrication of Holographic Sensors}

The photonic structures required for the generation of holographic sensors can be produced in several ways. Due to the 
optical properties being dependent upon the spacing of fringes, nanoscale fabrications are often required. This can range from casting an optical template to give a final product with optical properties or via the use of interferometric light interactions to stimulate regularly spaced changes within a substrate on a nanoscale. ${ }^{[51]}$ Within this review several of the most prominent and established fabrication methods will be examined.

\subsection{Surface Relief Gratings}

Holographic casting is a fabrication method that involves the copying of an optically active "master" template with a responsive hydrogel material. The resultant copy obtained from this process produces a device with chemical sensing properties alongside the optical characteristics of the template. The technique is advantageous as the production of optical devices can be relatively straightforward and low cost. ${ }^{[52]}$ Two main techniques are explored in this review, surface relief gratings and inverse opal structures. Surface relief gratings are where a responsive matrix solution is cast to copy a grating surface structure (Figure 3a). This process produces a grating structure on the exterior of the material which can interact with light as a simple wave guide. This fabrication method can be utilized with a wide range of substrates such as responsive monomers or responsive liquid crystals. ${ }^{[53]}$ The simplicity permits optical structures to be fabricated on large scale and offers opportunities for attachment to novel probes such as optical fiber for

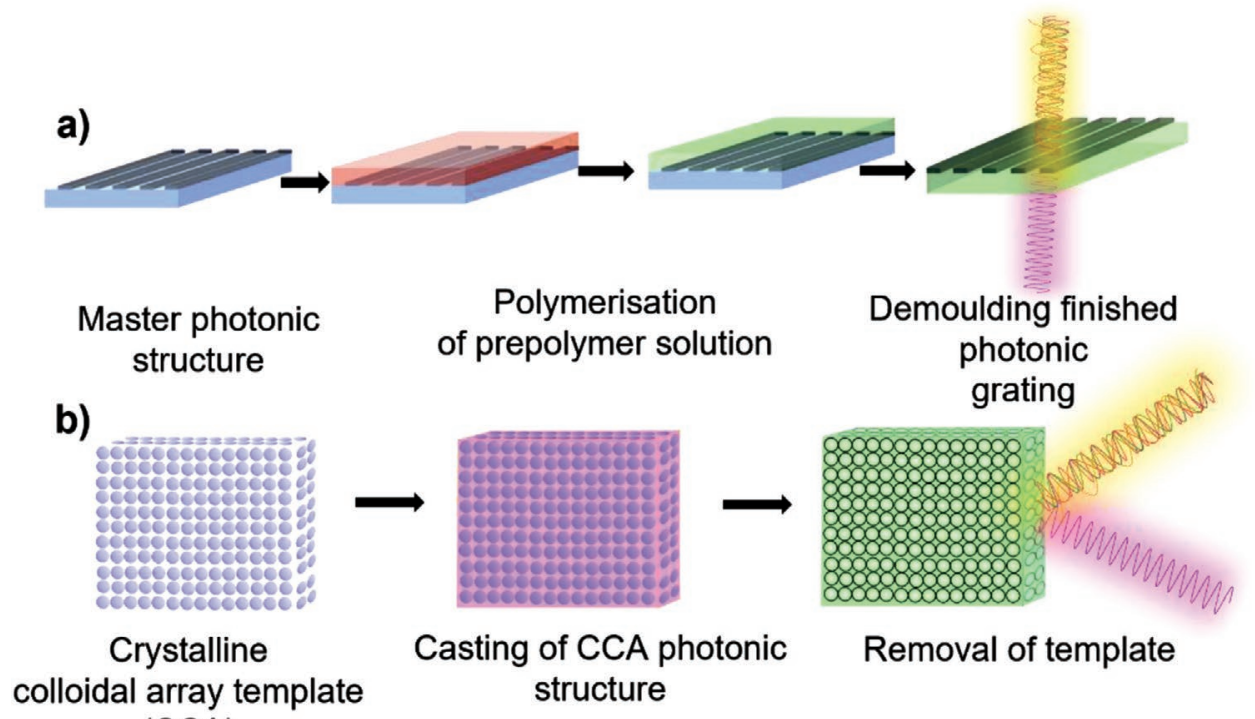

c) (CCA)
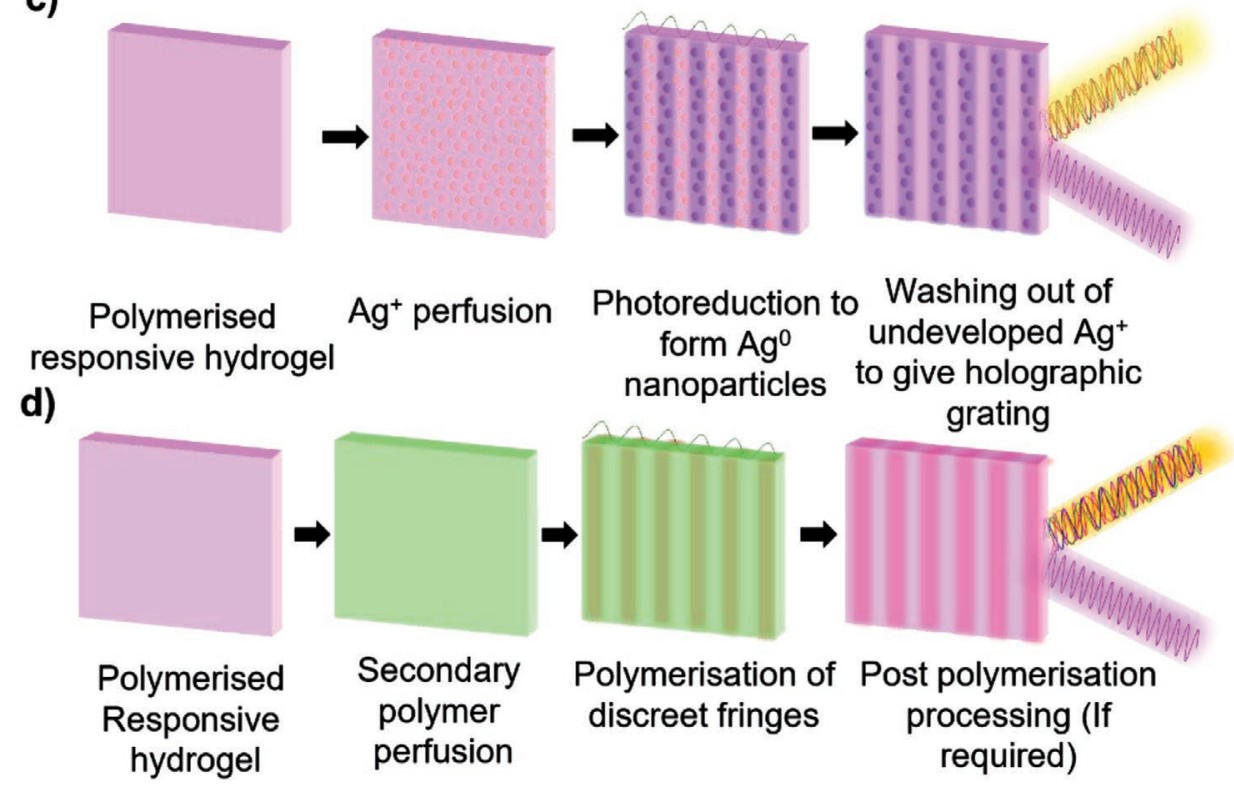

Figure 3. Fabrication techniques of holographic gratings. a) Photonic stamp casting of responsive hydrogels generating holographic surface gratings; b) Inverse opal fabrication via casting of a crystalline colloidal array; c) In situ photo reduction of metal salts to form metal nanoparticle fringes; d) Photopolymerization of secondary polymer fringes. 
medical devices. ${ }^{[54]}$ Sensors of this type have been demonstrated sensitivity to $\mathrm{pH}$, glucose, alcohols, ionic strength, ions, temperature, and humidity. ${ }^{[54-57]}$

\subsection{Inverse Opals}

Inverse opals differ from surface relief gratings due to the polymeric structure being a negative cast of a colloidal crystalline array (CCA) (Figure 3b). ${ }^{[5]}$ The CCA is responsible for imparting the photonic structure of the sensor, which can be fabricated through simple colloidal self-assembly. This simple master optical structure fabrication removes the requirement for expensive initial investment for a coherent laser source to generate the fringe structures therefore reducing overall costs of production. After polymerization, the CCA template can be dissolved generating an optical structure completely formulated from polymer. This is beneficial as the sensor does not possess any nanoparticles which permits simpler approval for use in biological systems..$^{[59]}$ The sensing properties are defined by the polymer used therefore the limitations on sensing are defined by the ability of hydrogels to respond to analytes. Inverse opals have been applied in a number of diverse fields such as $\mathrm{pH},{ }^{[60]}$ humidity, ${ }^{[61]}$ and macromolecules. ${ }^{[62]}$ Although the main focus in this review will be on the optical properties of inverse opal sensors, the unique morphology of the structures can be applied to controlled release and sorption of compounds in chemical applications due to their porous nature. ${ }^{[63]}$ Overall optical structure casting is a simple and powerful technique for producing optical sensors without the requirement for coherent laser light.

\subsection{Metal Nanoparticles}

Photolithographic production of metal nanoparticles is one of the most common methods used to fabricate holographic gratings. The process is comparable to development of silver nitrate film in black and white photography. To generate metal nanoparticle holograms, photochemical reactions are utilized to produce ultrafine layers of nanoparticles within a chemically responsive material (Figure 3c). Metal halide (i.e. AgBr) solutions are diffused into a chemically responsive material which is exposed to coherent laser light. ${ }^{[21]}$ Interference between incident and reflected laser light can generate points of high and low intensity to reduce metals such as silver. After photoreduction of metals ions and development stable nanoparticles are formed. Silver nanoparticles create a high change in refractive index producing a Bragg mirror structure within the polymer material. This production process is advantageous in several ways as the technique is reliable and gives rapid responses to chemical analyte changes. Nanoparticle holographic sensors have previously been applied in the detection of numerous stimuli, that is, glucose, enzymes, $\mathrm{pH}$, ions, ionic strength, and cocaine. ${ }^{[13,64-69]}$ Metal nanoparticle formulation methods were the genesis of photolithographic optical sensors and will be a corner stone of the field in future developments. In future with the development of novel nanoparticle/small molecule technology, ${ }^{[70,71]}$ nanoparticle holography could be functionalized with complex chemical properties.

\subsection{Nanoparticle-Free Holographic Sensors}

Techniques previously described for the fabrication of holographic structures depends upon materials of inorganic materials or physically printed fringes to generate changes within a material to vary the refractive index. Another photolithographic method is the use of a secondary polymeric material to generate holographic structures. ${ }^{[72]}$ To fabricate nanoparticle free sensors, a monomer solution with a high crosslinking concentration is soaked into the sensitive hydrogel matrix. Once saturated the hydrogel is exposed to laser light, where constructive and destructive interference between incident and reflected beams polymerize fringes at points of high intensity producing a grating structure. Variation of refractive index is achieved by the increased crosslinking density to produce a wavelength selective Bragg mirror within a responsive hydrogel (Figure 3d). At present nanoparticle free holograms have been applied in sensing alcohol, glucose, ions, temperature, and humidity. ${ }^{[72-75]}$ The removal of nanoparticles from holographic gratings removes some of barriers that have previously hindered the nanoparticle-based systems due to the potential health risks of nanoparticle leaching. ${ }^{[76]}$ Benefits are also observed in the reduction in the complexity of processing of holograms, by removing the development and fixation steps required in metal nanoparticle holographic sensors the production of holographic gratings on a mass scale is more achievable. These chemical processes also require the use toxic chemical reagents which can be carried over into the finished product, by removing these steps entirely the potential risks are mitigated. The improved scalability can also be attributed to the reduction in cost of raw materials, nanoparticle free holograms only require monomer compounds to create holographic gratings and do not require the use of silver and gold, two of the most precious elements on the planet. Overall nanoparticle free holographic sensors are not as well established as nanoparticle-based systems but with time these systems could allow for greater expansion. An array of the applications covered in this review are listed in Figure 4.

\section{Applications of Holographic Sensors}

Holographic sensors have been expanding at a rapid rate improving the selectivity and sensitivity to compete with that of conventional biosensing techniques. This has been achieved with techniques such as the molecular imprinting of hydrogel materials and increasing the selectivity of chemical binding sites through specific chemical interactions. Throughout this section we aim to demonstrate the recent developments in holographic optical sensors, highlighting the improvements in selectivity, sensitivity, and simplicity of readout.

\section{1. $\mathrm{pH}$ Monitoring}

Monitoring of $\mathrm{pH}$ is universal across all physiological processes, $\mathrm{pH}$ indicates respiratory issues, ${ }^{[77]}$ wound healing, ${ }^{[78]}$ and diagnosis of gastro oesophageal reflux disease. ${ }^{[79]} \mathrm{A} \mathrm{pH}$ sensor was fabricated in the form of 1D slanted optical crystal flakes. ${ }^{[80]}$ The fabrication process involved preparing the monomer solution 


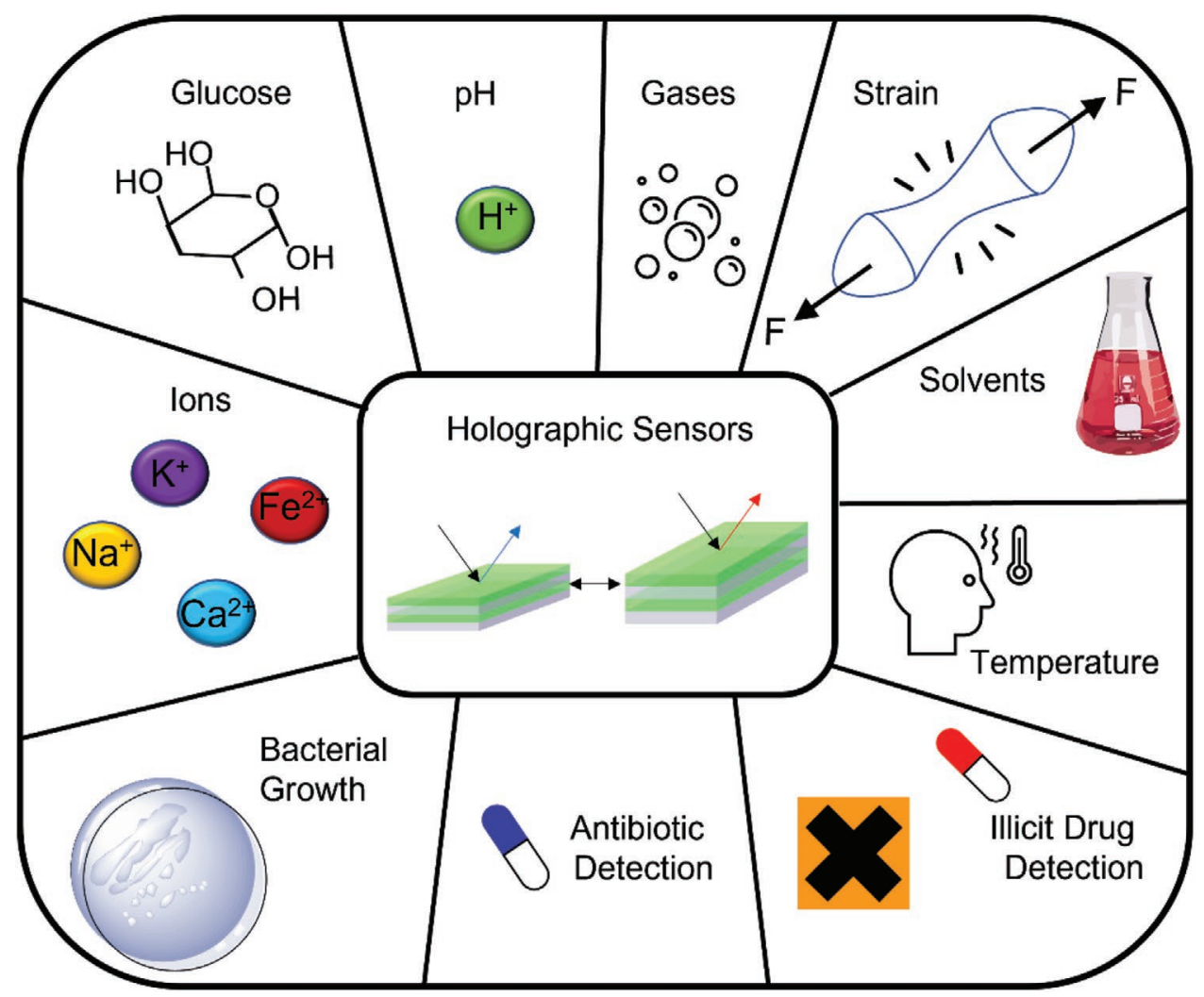

Figure 4. Summary of the analytes to be covered throughout this review.

consisting of HEMA (91.5 mol\%), EGDMA (2.5 mol\%), and methacrylic acid (MAA) (6 mol\%), which was then polymerized using free radical UV polymerization on PMMA surface (chosen as a temporary substrate). $\mathrm{AgNO}_{3}$ salt was used as a source of $\mathrm{Ag}^{+}$for embedding $\mathrm{Ag}^{0}$ NPs in the p(HEMA-co-MAA) matrix which was exposed to a $5 \mathrm{~ns}$ Nd:YAG pulsed laser $(\lambda=532 \mathrm{~nm}$, $350 \mathrm{~mJ}$ ) (Figure $5 \mathrm{a}$ ). The sample was immersed in the exposure bath at an inclination of $5^{\circ}$ from the surface plane of the mirror (Figure 5b). The film was detached from the PMMA substrate via immersion in a water/ethanol solution $(50 \% \mathrm{v} / \mathrm{v})$, where films would detach to be subsequently shaped into round PC flakes.

The $\mathrm{pH}$ response of the sensor is based on ionization of the carboxylic acid groups in the hydrogel matrix as the $\mathrm{pH}$ increases. As they ionize, the Donnan osmotic pressure increases, resulting in water uptake into the p(HEMA-co-MAA) matrix, increasing $\mathrm{Ag}^{0} \mathrm{NP}$ lattice spacing, shifting the Bragg peak into longer wavelengths. The grating was illuminated with a supercontinuum white light laser and the backscattered light was documented. When the $\mathrm{pH}$ was increased from 4.0 to 8.0, the Bragg peak of the PC flakes shifted from 510 to $620 \mathrm{~nm}$, which was accompanied by color change (Figure $5 \mathrm{c}, \mathrm{d}$ ). This shift corresponded to a $\mathrm{pH}$ sensitivity of $0.1 \mathrm{pH}$ units. The Bragg peak shift by free-standing flakes was about half of the Bragg shift by the $\mathrm{pH}$ sensors attached to the PMMA substrate. This could be attributed to the flakes having a greater degree of freedom to expand than the attached substrate, but further investigation would be required to confirm this hypothesis. The sensor offers several advantages, such as label free detection, qualitative measurements, and immunity to photobleaching.
The fabrication method presented in this investigation allows creating $\mathrm{pH}$ sensors at mass scale. The volumetric change caused by change in $\mathrm{pH}$ was found to be reversible, giving the sensor the potential for reusability. Moreover, the pHEMA matrix can be functionalized to tune the $\mathrm{pH}$ sensing range.

\subsection{Ion Sensor}

Surface relief gratings have long been hoped to offer a cheap and reliable alternative to ion concentration determination in biological samples. Naydenova et al. demonstrate a methodology whereby holographic surface relief structures are formed and coated in an ionophore containing polyvinyl chloride (PVC) matrices. ${ }^{[81,82]}$ The methodology described within the paper illustrates that the grating is initially formed utilizing an Nd:YAG laser which is separated and directed onto the photopolymer surface. To sensitize the grating structure a secondary layer is spin coated onto the surface containing the binding ligand dibenzo-18-crown-6 (DC) or tetraethyl 4-tertbutylcalix[4]arene (TBC) PVC and plasticizers to give rise to a sensitized coating. These two sensitizing units are ionophores expected to show selective binding for $\mathrm{K}^{+}$and $\mathrm{Na}^{+}$respectively. Volumetric changes in this outer coating tunes the periodicity of the grating, altering the gratings diffraction intensity.

DC having a significantly larger ring structure has a higher binding affinity for $\mathrm{K}^{+}$ions due to their larger ionic radii, this conclusion is confirmed by the results obtained within the article. Where the alteration in diffraction efficiency for 
a)

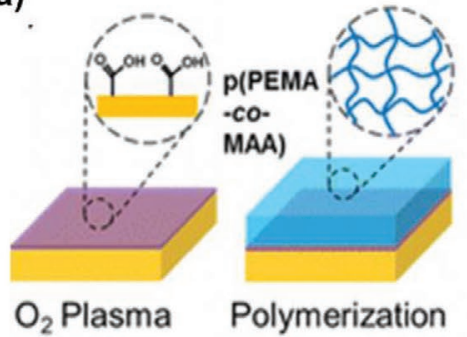

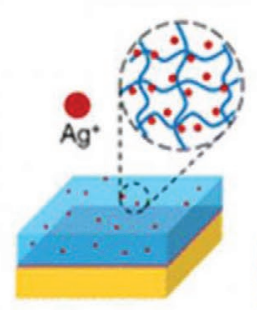

Ion Doping

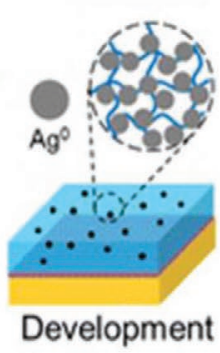

b)

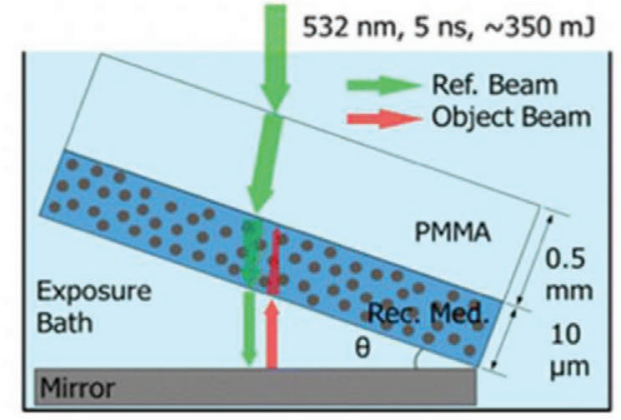

c) i)

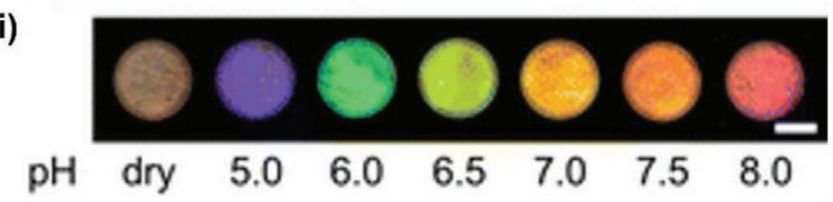

ii)

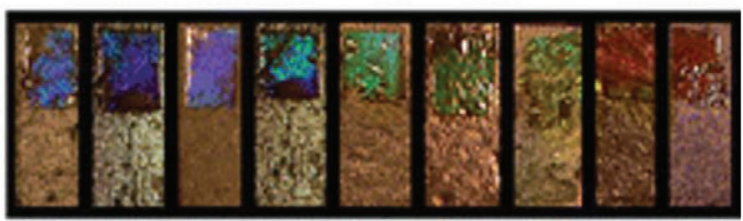

$\mathrm{pH} \begin{array}{lllllllll}3.0 & 4.0 & 5.0 & 5.5 & 6.0 & 6.5 & 7.0 & 7.5 & 8.0\end{array}$

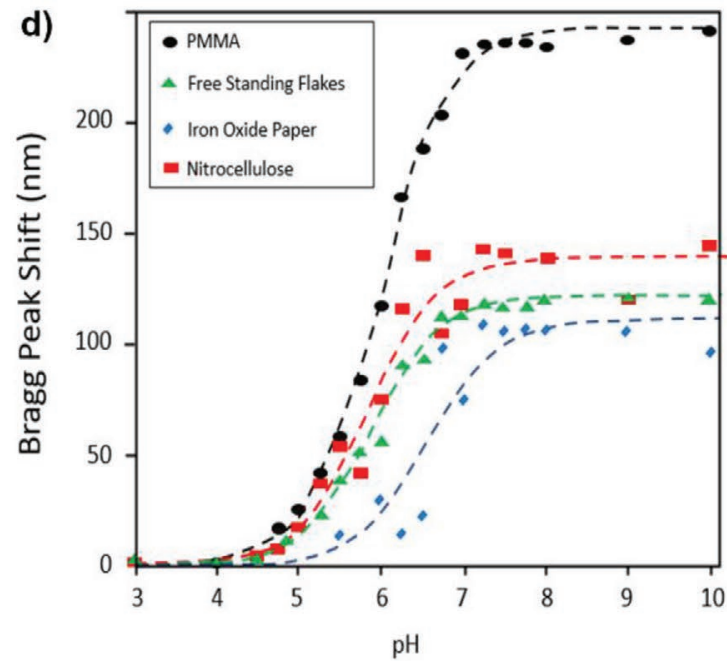

Figure 5. Overview of MAA modified film pH responses. a) Schematic of sensor preparation; b) Schematic of laser writing of 1D PC matrix; c) Photographs of i) free-standing flakes and ii) paper backed flakes in response to $\mathrm{pH}$ range $5.0-8.0$; $\mathrm{d}$ ) Bragg peak shifts as a function of $\mathrm{pH}$ in $\mathrm{pH}$ sensors of different forms. Reproduced with permission. ${ }^{[80]}$ Copyright 2018 , American Chemical Society.

DC decreases from 1 to 0.6 for $\mathrm{K}^{+}$whereas for $\mathrm{Na}^{+}$the drop is significantly reduced to 1 to 0.9 . The reverse was observed for TBC, the $\mathrm{Na}^{+}$response observed the diffraction decrease from 1 to 0.52 , in comparison results obtained for $\mathrm{K}^{+}$of 1 to 0.8 . For application in a medical device the technology shows real promise and could be a true asset to the monitoring of ionic concentrations within the body. Although the methodology is promising and there is further work required to bring the sensitivity of the device to utilizable levels, it is a novel demonstration that there is capability of optical sensors to begin to rival the conventional devices in use today.

Yetisen et al. report a nanoparticle free methodology for selectively sensing $\mathrm{Cu}^{2+}$ and $\mathrm{Fe}^{2+}$ utilizing a pulsed laser reflection set up. ${ }^{[7]}$ Deficiencies in $\mathrm{Fe}^{2+}$ and $\mathrm{Cu}^{2+}$ in blood serum can be an indication of chronic illnesses, such as Wilsons disease and anaemia. ${ }^{83,84]}$ The research demonstrates the way in which chemical stimulus can alter the fringe spacings between areas of high and low refractive index within hydrogel networks leading to a change in the reflection spectrum observed (Figure 6a). This development removes several matters that have hindered the utilization of silver halide nanoparticles in previous years. Inconsistent diffusion of $\mathrm{Ag}^{+}$ions into the hydrogel film prior to exposure decreases the sensitivity of grating significantly, due to disparity in diameter and deposition of NP's. ${ }^{[74]}$ This is also paired with the problem that often $\mathrm{Ag}^{+}$ions can interfere with a variety of sensitizing agents prior and post fabrication. Yetisen et al. describe a methodology which removes the requirement for Ag NP by utilizing a functionalized crosslinking photosensitive monomer (Figure 6b) ${ }^{[74]}$ By undertaking a condensation reaction between pyrrole and 4-formylbenzoic acid, a porphyrin structure was synthesized. To incorporate into the polymer films modification of the sensitizing agent with 3-(4-hydroxyphenoxy)propyl acrylate via an esterification reaction inserts a double bond unit capable of undergoing a free radical polymerization reaction. The porphyrin ring synthesized has free amine lone pairs in an orientation capable of binding ions selectively. The ring structure when incorporated into holographic gratings showed selectivity for $\mathrm{Cu}^{2+}$ and $\mathrm{Fe}^{2+}$, with quantitative colorimetric readouts (Figure $6 c, d$ ). By integrating this functional porphyrin ring into a pHEMA matrix and utilizing a pulsed laser $(350 \mathrm{~mJ}, 532 \mathrm{~nm}$, $6 \mathrm{~ns})$, a Bragg reflection grating was formulated with a replay wavelength within the visible region. Binding of ions adjusts the fringe spacings within the film generating a corresponding change in the reflected wavelength. This research has been able to demonstrate the selective binding of ions but also a nanoparticle free path to a new generation of holographic sensors which can remove several barriers such as cost and limited functionality creating opportunities for their widespread application. 
a)

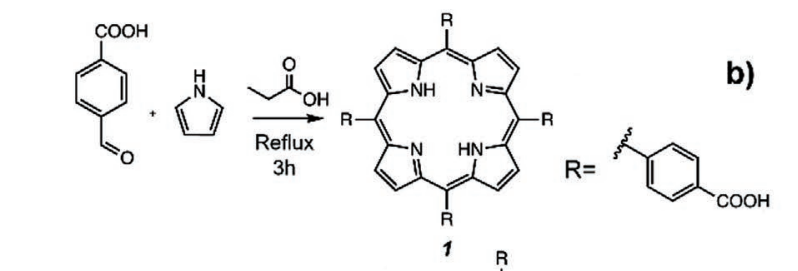

b)

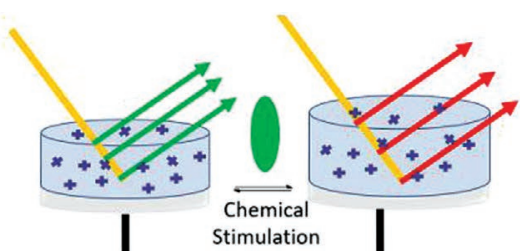

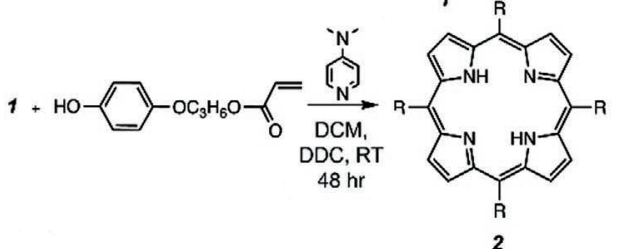

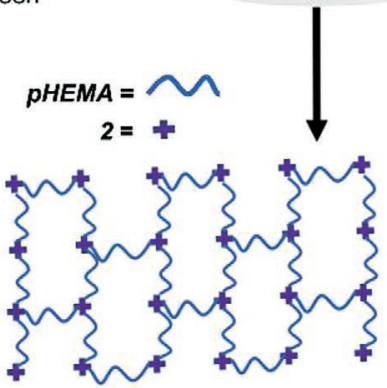

$\mathrm{R}=$

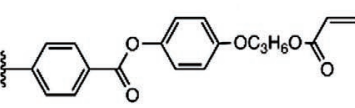

d)
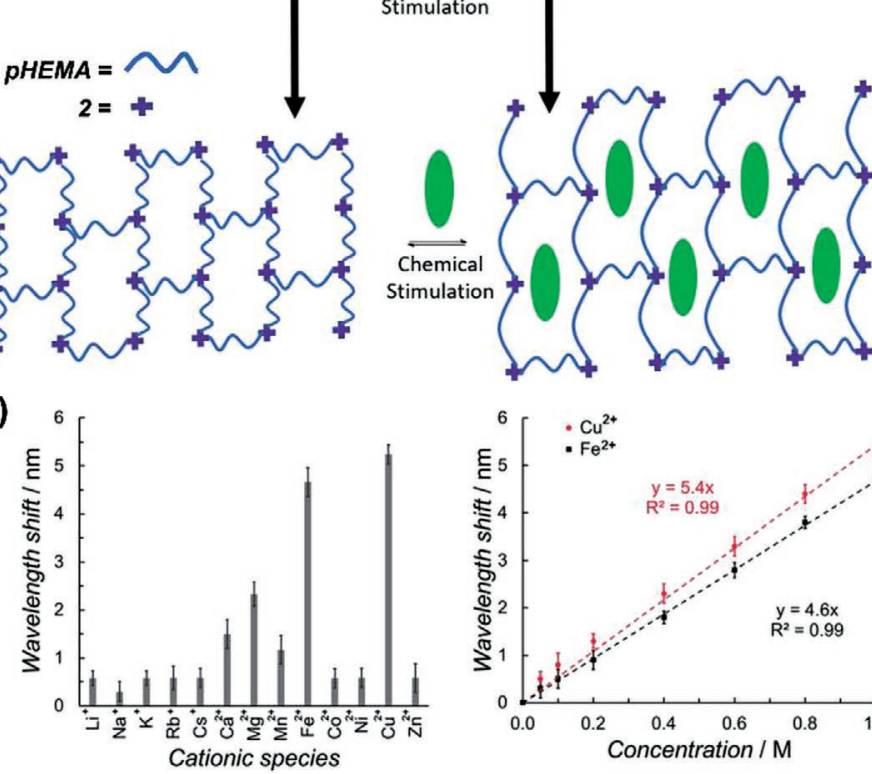

c) i)
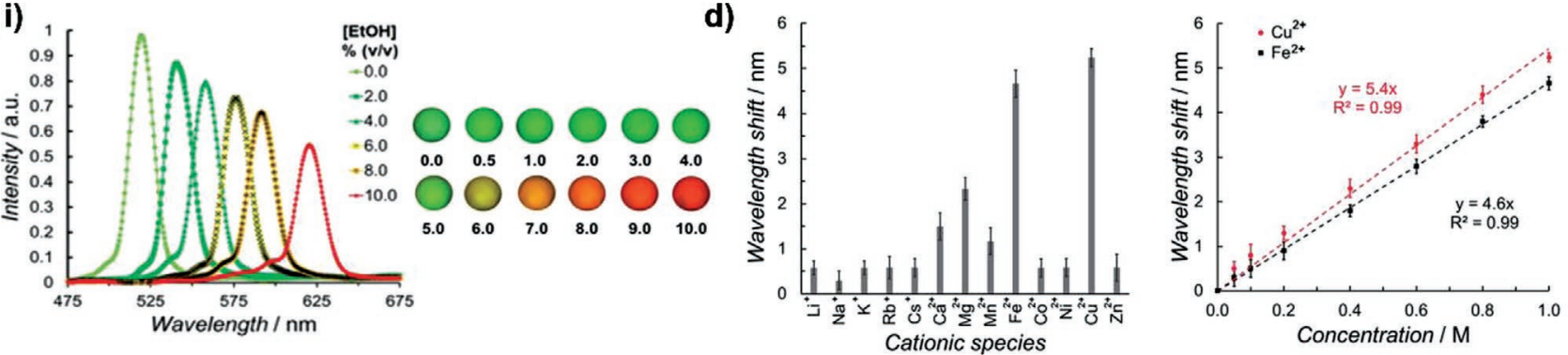

Figure 6. Selective nanoparticle free holographic $\mathrm{Cu}^{2+}$ and $\mathrm{Fe}^{2+}$ sensor. a) Schematic to demonstrate the response and reversible expansion of the hydrogel films upon chemical stimulation; b) Synthetic pathway in the fabrication of porphyrin ring structure capable of binding metal ions; c) Graphs showing the wavelength shifts observed for the holographic metal ion sensor to different alcohols; d) Graphs illustrating the selective binding of Fe ${ }^{2+}$ and $\mathrm{Cu}^{2+}$. Reproduced with permission. ${ }^{[74]}$ Copyright 2016, Royal Society of Chemistry.

\subsection{Temperature and Humidity Sensing}

With the further expansion of the exploration of photopolymers in the preparation of holographic gratings, there have been interesting developments in the hybridization of printed photopolymer structures with a variety of sensing components. The formation of composite materials allows for greater functionalization of biosensors with alternative functionalities. ${ }^{[85]}$ By employing zeolites within the hydrogel network, alternative functionalization's can be achieved. Naydenova and Mintova et al. have demonstrated the utilization of the hydrophilic and hydrophobic properties of zeolite nanocrystals in the inkjet printing of alcohol sensitive holographic gratings (Figure 7a). ${ }^{[86]}$ In the article there is description of the formulation methodology for both transmission and reflection holograms, the method differs but the components utilized do not vary. The presence of zeolite nanocrystals is to create a change in refractive index between differing layers of the holographic grating, this leads to requirement for particles of homogenous particle diameter. Disparity in diameter produce irregularities with diffusion rates, optical properties, and creates a larger surface area which reduces the reliability of measurements. The inkjet printing of zeolite nanocrystals onto photo polymer films to produce films is beneficial in the production of reflection holograms, but this formulation method does not lead to significant benefits in the production of transmission holograms as current technology does not allow for sufficient resolution or accuracy to produce gratings capable of interacting with light effectively. To generate transmission holograms zeolite nanocrystals are dispersed within the base polymer film, then on to this a holographic grating is recorded creating areas of differing refractive index are created.

Two different crystalline structural conformations of zeolite nanocrystal, MFI and EMT, are highlighted within this work. The main difference in these two arrangements is the channel formation, MFI conformation bares channels running straight through the material and an EMT conformation where the flow of analyte is obstructed. These properties make the two structurally different materials ideal for different purposes, MFI for reversible measurements and EMT for non-reversible. The capability of these printed zeolite nanocrystalline holograms in the irreversible detection of analytes such as $\mathrm{H}_{2} \mathrm{O}$ is highlighted. By depositing zeolite nanocrystals in regularly patterned concentric circles with the highly hydrophilic EMT structured zeolites. The sensors initially display a red color but after an annealing process lose visible color due to the removal of water present in the formulation process. Removal of water diminishes the fringe spacings to a shorter wavelength outside of the visible spectrum. Upon exposure to a humid environment the zeolite nanocrystals will take up water leading to a change in the fringe spacings and therefore the observed color (Figure 7b). The highly hydrophilic nature of the EMT structure leads to the capturing of water that could not be removed even when exposed to low humidity sequentially. The irreversibility of the sensor response allows users to identify the occurrence of changes in humidity between monitoring checks. 
a)

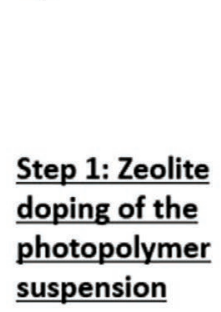

Photopolymer suspension

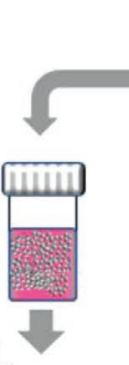

Step 2: Zeolite-doped photopolymer deposition

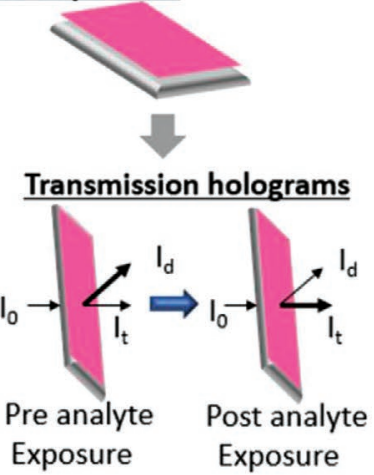

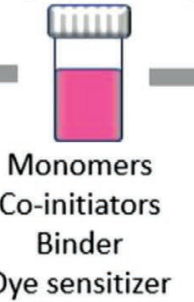

Dye sensitizer
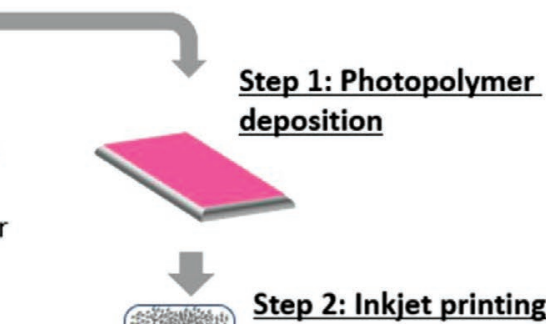

Step 2: Inkjet printing of nanozeolites Defined pattern of nanozeolites

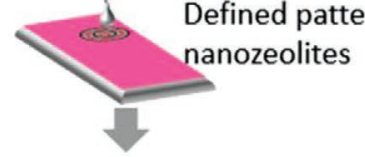

Reflection holograms

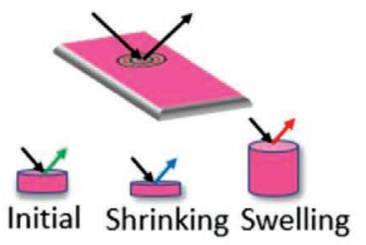

b)

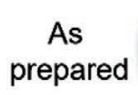

As
prepared

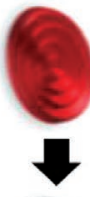

Annealed

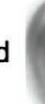

c)
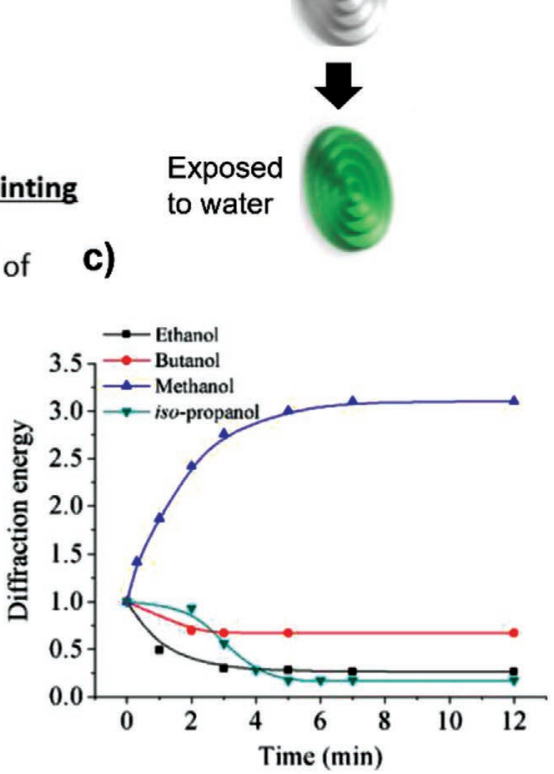

Figure 7. Overview of inkjet printed zeolite nanoparticle functionalised holographic gratings for humidity determination. a) Fabrication of zeolite nanoparticle based holograms; b) Visual response of holographic films pre and post exposure to water; c) a graph to display the response of sensors when exposed to various simple short chained alcohols. Reproduced with permission. ${ }^{[86]}$ Copyright 2018, American Chemical Society.

The sensors developed were designed to test for the presence of alcohols, the testing was carried out within a gas chamber where the respective alcohols were in the gas phase. A specificity for methanol was shown over ethanol, isopropanol, and butanol, where the diffraction efficiency decreased by $0.2,0.2$, and 0.75 respectively except for methanol which increased by a factor of 3.2 (Figure 7c). This is hypothesized within the paper to be due to two different factors, the ability to be taken up by the zeolite crystals and the nature of the swelling. The expected response of the nanocrystals is for a decrease in the diffraction efficiency as is observed for the longer alkyl chained alcohols. The response observed is caused by the increased uptake of the methanol due to its shorter alkyl chain giving a significantly increased change in comparison to the other alcohols and due to the highly polarity of methanol giving an opposing response in the hydrophilic nanocrystals.

A holographic sensor developed for detecting changes in temperature and relative humidity. ${ }^{[75]}$ This investigation explores various photopolymers for reversible sensing of relative humidity and temperature, as well as possibility of developing humidity and temperature stable sensors. Sensor response can be modified via polymer composition variation. Three photopolymer layers were studied, in transmission and reflection mode. Photopolymer layers were prepared by deposition of photosensitive solutions onto glass slides. Volume phase transmission and reflection gratings were recorded using a $\mathrm{Nd}: \mathrm{YVO}_{4}$ laser (532 nm) (Figure 8a-c). Afterwards, to polymerize all residual monomers, gratings were exposed to UV light. The humidity response was studied by determining the diffraction efficiency of transmission gratings and the reflection gratings spectral response of as relative humidity was varied. A controlled humidity and temperature chamber was utilized to accurately establish distinct humidity levels whilst maintaining constant temperature. Differential scanning calorimetry (DSC) was used to analyze the moisture loss from the photopolymer layers under heating. For the transmission gratings, the change in diffraction efficiency was measured by recording Bragg selectivity curves in $\mathrm{RH}$ range $20-90 \%$ and normalized to diffraction efficiency at $\mathrm{RH}=20 \%\left(\eta_{20}\right)$. Figure $13 \mathrm{~d}$-i demonstrates that the diffraction efficiency variations of photopolymer $\mathrm{C}$ were very significant, that is, increase of the diffraction efficiency from $6 \%$ to $84 \%$ was observed as the relative humidity changes from $20 \%$ to $90 \%$. Little change was shown by photopolymers $\mathrm{A}$ and B. Reversibility of the humidity induced changes was confirmed for $\mathrm{RH}$ up to $70 \%$ for photopolymer A, up to $90 \%$ for photopolymer B and up to $60 \%$ for photopolymer C. Measuring reflection gratings response shift in relative humidity was carried out by monitoring the peak intensity and position in the spectral response over a range of $20-80 \% \mathrm{RH}$ and normalizing to the wavelength of the diffracted light at $\mathrm{RH}=20 \%$ $\left(\lambda_{20}\right)$. In Figure $8 \mathrm{~d}$-ii, photopolymer A demonstrates a nonlinear response. Photopolymer B is comparatively unresponsive to variations of $\mathrm{RH}$ below $60 \%$ but displays an almost linear response at $\mathrm{RH}>60 \%$. Photopolymer $\mathrm{C}$ displays significant 


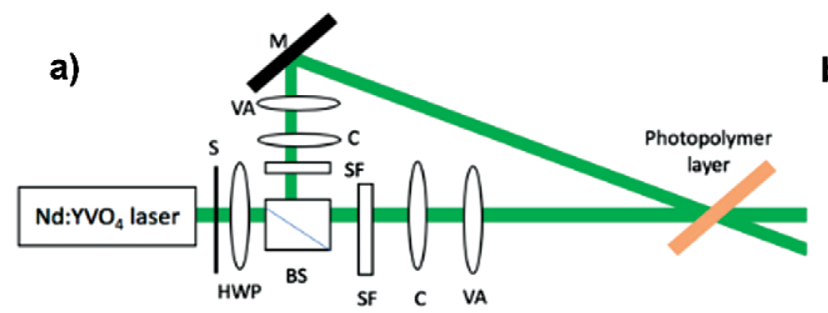

b)

c)
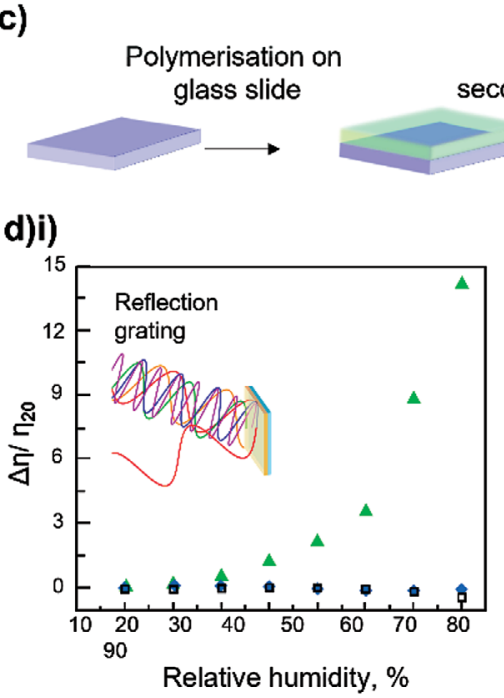

ii)

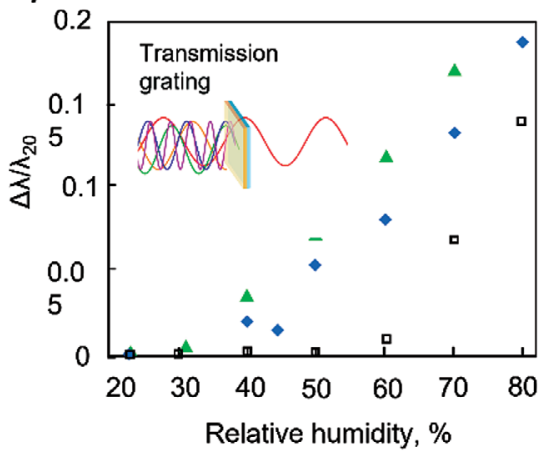

Soaking of condary polymer
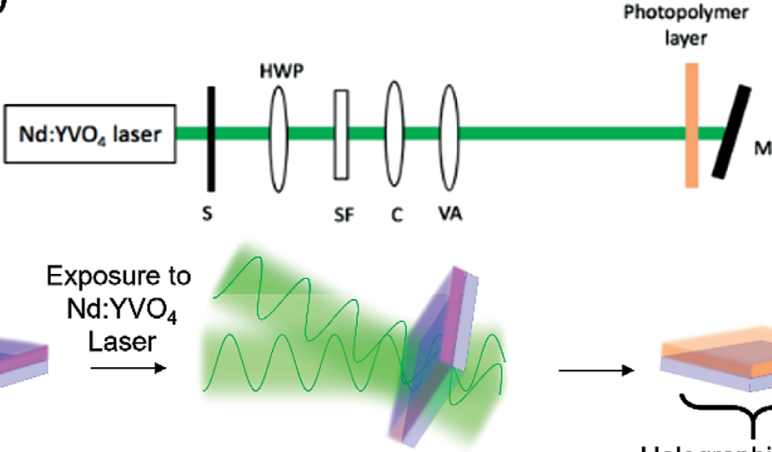

ii)

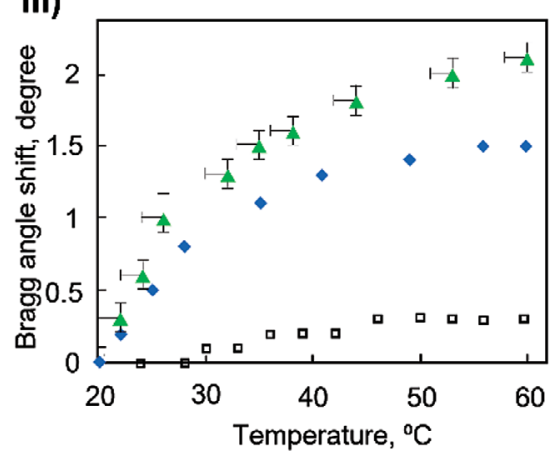

Figure 8. Experimental recording set-up for a) slanted transmission grating and b) slanted reflection grating; $\mathrm{S}$ - electronic shutter; HWP - half-wave plate; BS - beam splitter; SF - spatial filter; C - collimator; VA - variable aperture; M - mirror; c) Schematic of the holographic grating fabrication; d) i) Relative changes in the on-Bragg diffraction efficiency of slanted transmission gratings recorded in photopolymers versus relative humidity; ii) Spectral response of reflection gratings recorded in photopolymers versus relative humidity; iii) Bragg angle shift temperature dependence of slanted transmission gratings recorded in photopolymers. Photopolymers: photopolymer A ( $\bullet$ ), photopolymer B $(\square)$ and photopolymer C $(\boldsymbol{\Delta})$. Reproduced with permission. ${ }^{[75]}$ Copyright 2017, Elsevier B.V.

sensitivity, especially within the range of $40-70 \% \mathrm{RH}$. The shift of the maximum intensity in the spectral response of each photopolymer layer was observed to be reversible to within $4.8 \pm 2 \mathrm{~nm}$. The humidity response of the photopolymers can be attributed to their permeability to water vapor. Photopolymer $\mathrm{C}$, with the most hygroscopic compounds, shows biggest changes in the optical properties of the holographic gratings. The distinct responses of photopolymer layers can be explained by their capability to expel water due to increase in temperature, biggest in photopolymer $\mathrm{C}$, which experiences the most shrinkage. Since photopolymer $\mathrm{C}$ is also responsive to humidity, temperature response of sealed layers was also investigated to eliminate effects due to humidity and only account for the thermal effects. The temperature response of slanted transmission gratings was investigated in $20-60^{\circ} \mathrm{C}$ range. In photopolymer $\mathrm{C}$, exposure to temperature in this range causes contraction of the layers and Bragg angle change up to $2^{\circ}$ (up to $1.5^{\circ}$ for $\mathrm{A}$ and $0.3^{\circ}$ for $\mathrm{B}$ ).

\subsection{Strain Sensor}

The accurate determination of strain is complex due to the requirement for complex laser-based systems to monitor minor deformations in a structure such as buildings, aircrafts, and bridges which can lead to catastrophic failures. ${ }^{[87-89]}$ Strain sensors are of great importance with a number of both hydrogel and holographic strain sensors have been demonstrated in recent years using a range of fabrication techniques. ${ }^{\text {[9-92] }}$ AlQattan et al. developed a holographic strain sensor through use of holographic interference patterning to record curved nanostructures on adhesive tapes, which upon deformation vary their diffraction properties. ${ }^{[93]}$ Common black ink (Staedtler Lumocolor) deposited onto a glass substrate was utilized as the recording medium. Holographic gratings were produced using an Nd:YAG laser system creating either Fresnel lens or a curved grating nanostructure by controlling the point of reflection from the concave mirror, with grating spacing tuned through the variation of recording angle (Figure 9a). The gratings recorded could interact with broadband light to generate a rainbow diffraction pattern, by varying the angle of illumination the degree of diffraction increased, allowing researchers to identify a variation in the angle relative to the normal (Figure 9b). In addition to these rotational experiments, by adhering the gratings to a tape $(4 \mathrm{~cm})$ substrate the effect of strain could be studied. The tape bearing the holographic grating was stretched over 300 increments ( $5 \mu \varepsilon$ per stretch), giving a total expansion of $0.6 \mathrm{~cm}$. Increasing the distance stretched lead to an increase in the level of diffraction. This correlated with stretching elongating the fringe spacing within the sensor, this variation in diffraction efficiency could be correlated with the level of strain. The sensors developed within this work showed little vulnerability to interference from water or $\mathrm{pH}$ solution due to the 


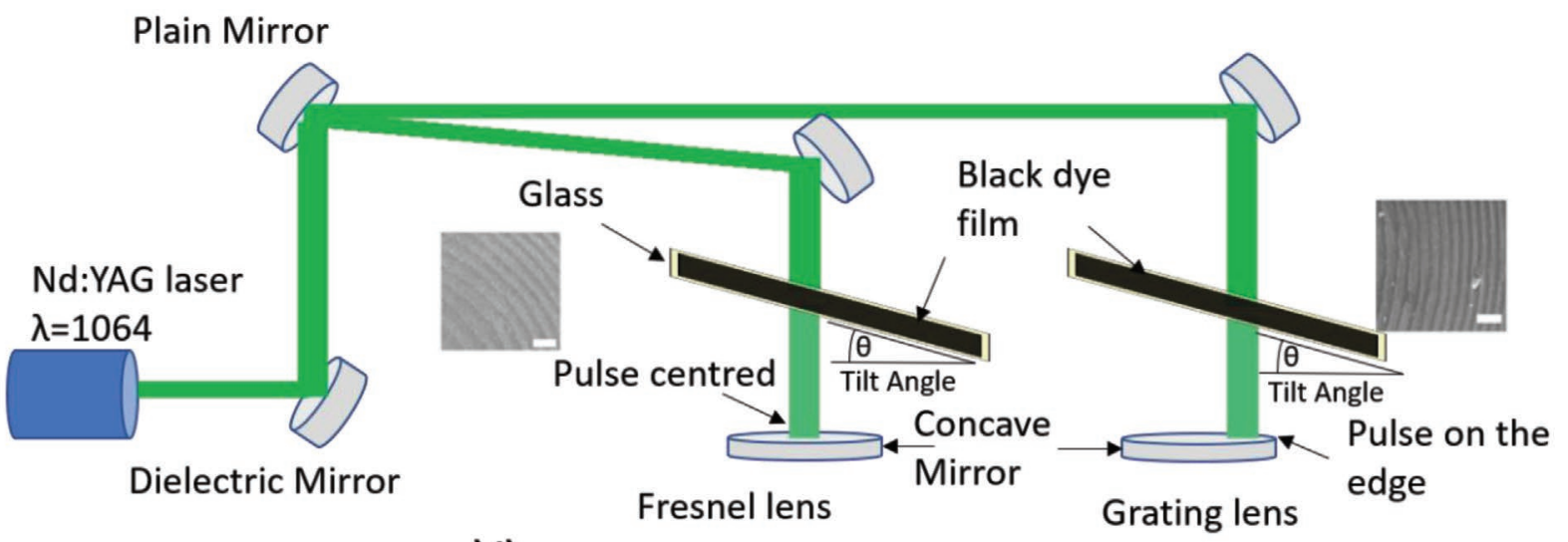

b) i)

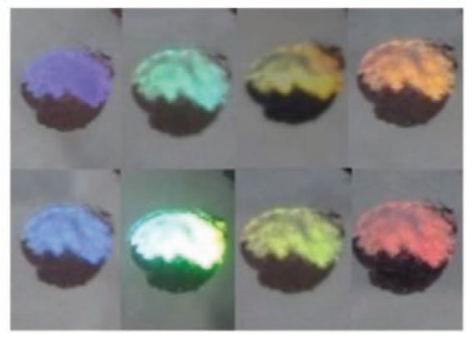

c) i)

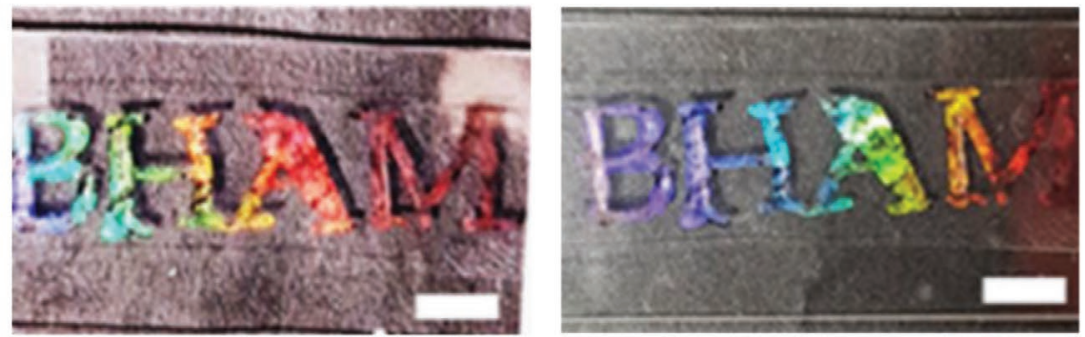

Figure 9. Black dye diffraction gratings for the identification of strain; a) Fabrication set up for the production of either Fresnel or grating lenses dependent on the point of reflection from a concave mirror inset SEM images of Fresnel lens and Grating lens respectively, scale bar $100 \mu \mathrm{m}$; b) colourimetric response of sensors upon rotation of the diffraction grating; c) Nanograting of BHAM logo to demonstrate the images recordable and the variation under strain. Reproduced with permission. ${ }^{[93]}$ Copyright 2019, American Chemical Society.

hydrophobicity of the ink utilized. Low wettability is vital when monitoring stress in real world situations such due to rain or external influence. Alongside the lack of interference these sensors are easily fabricated from simple materials, a readily available ink and a simple substrate of glass or tape, this allows for low cost and easily accessible. The fabrication method could be easily modified to generate images in the gratings, such as lettering illustrated in Figure 9c which was responsive under strain.

\subsection{Solvent Sensor}

Elsherif et al. developed holographic optic fiber sensors responsive to alcohols at $0-50$ vol\% and $\mathrm{pH} .{ }^{[94]}$ The hydrogel sensors were prepared from monomer solutions consisting of 2-hydroxyethyl methacrylate (HEMA) and ethylene glycol dimethacrylate (EGDMA). Acrylic acid was added as a co-monomer for hydrogel functionalization in the $\mathrm{pH}$ sensor (Figure 10a). For the hydrogel optical fibre fabrication, ethylene glycol diacrylate (EGDA) was mixed with 2-hydroxy-2-methylpropiophenone (HMPP) in DI water. The prepared solution $(200 \mu \mathrm{L})$ was injected into a polyvinyl chloride tube and polymerized under UV light generating a flexible optical cable. Affixing of the holographic grating was achieved via silanization of the tip and submerging in either alcohol or $\mathrm{pH}$-sensitive monomer solutions that were pipetted on asymmetric micro lens arrays and the tube was UV cured. The sensors were tested in both of transmission and reflection modes (Figure 10b), either using a smartphone or an optical power meter. Output signals meas- ured via spectrophotometer validated smartphone readout as a viable, simple, and accessible analysis technique.

The alcohol-responsive sensor was tested for response to ethanol, propan-2-ol, and dimethyl sulfoxide (DMSO). In both modes, the highest sensitivity was with respect to DMSO. The sensor responded to all three analytes in the range 0-50 vol\% with the limit of detection of $2 \%$ and was only linear for propan-2-ol. The trends observed in both of transmission and reflection modes were similar, although the sensitivities in reflection mode were significantly smaller than in transmission mode (Figure 10c). The pH-responsive sensor showed good sensitivity in $\mathrm{pH}$ range $5.0-7.0$ but a diminished response at $\mathrm{pH}$ higher than 7.0 (Figure 10d). Sensitivity was decreased in reflection mode, which could be attributed to the readout setup and the angle of illumination of the sensor. The approach presented offers a real-time sensing method with simplistic readouts and fabrication methods. The sensor can be fabricated using biocompatible fibers, which improves suitability for in vivo applications and the optical sensing mechanism is immune to electromagnetic interference occurring in common diagnostic techniques such as MRI scans. Relatively minimal analyte volumes ( $1 \mathrm{~mL}$ for sensor attached on the glass slide) are required for analysis. The sensors are highly reproducible and have a rapid equilibration time $(60 \mathrm{~s} \pm 10 \mathrm{~s}$ for $\mathrm{pH}$ sensor and approximately $60 \mathrm{~s}$ for alcohol sensor, depending on alcohol type and concentration). Sensors could be modified to show sensitivity at higher $\mathrm{pH}$ and enhance the sensitivity of biocompatible fiber systems.

By employing a nanoparticle free fabrication method Jiang et al. formulated a holographic alcohol sensor capable of determining the concentrations of methanol, ethanol, 1-propanol, 
a)

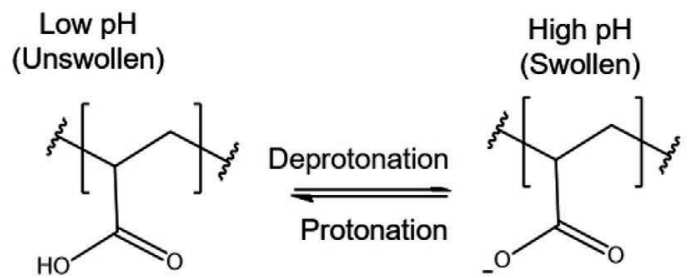

c) i)

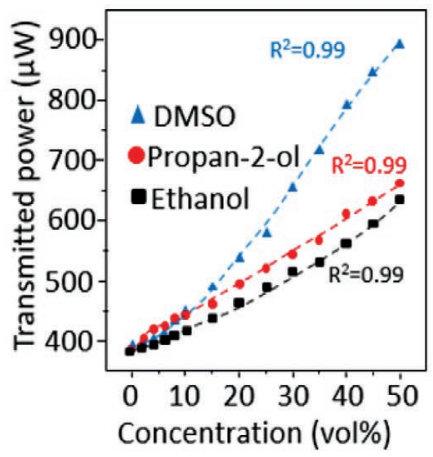

ii)

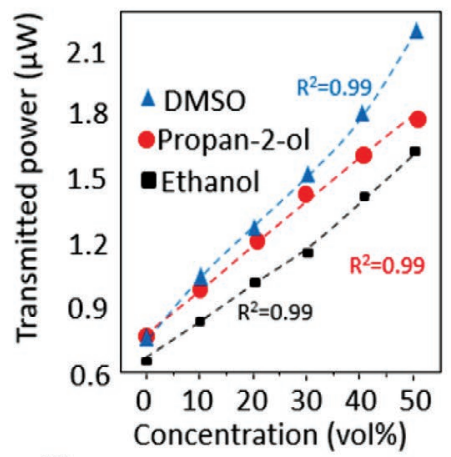

ii)

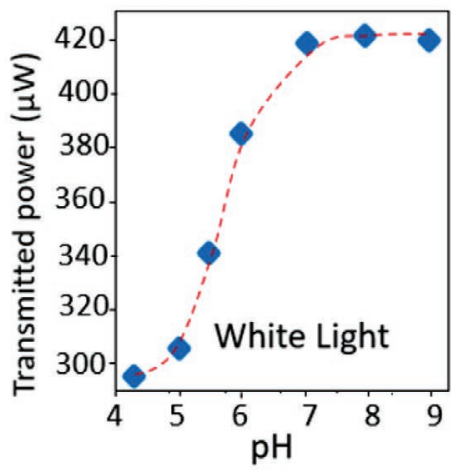

Figure 10. The $\mathrm{pH}$ and alcohol responsive films fabricated by surface relief casting. a) Equilibrium reaction to initiate the swelling and shrinking of the hydrogel film; b) Setup for testing in i) transmission mode and ii) reflection mode; c) Interrogation of the fibre optic probe for alcohol sensing in the transmission and reflection configurations: i) The maximum optical transmitted power versus alcohol concentrations; ii) The reflected power versus alcohol concentrations; d) Interrogation of the fibre optic probe for $\mathrm{pH}$ sensing in the transmission and reflection configurations: i) The maximum transmitted power versus $\mathrm{pH}$; ii) The reflected power versus $\mathrm{pH}$. Reproduced with permission. ${ }^{\text {[94] }}$ Copyright 2019, Royal Society of Chemistry.

and isopropanol via UV photopolymerization of a secondary highly crosslinked polymer. ${ }^{[73]}$ By utilizing in situ photopolymerization the requirement addition of dyes such as Safranin $\mathrm{O}$ is removed. ${ }^{[72]}$ The base hydrogel matrix consists of a loosely crosslinked hydrogel matrix of HEMA (93.9 mol\%), glycerol dimethacrylate (4.2 mol\%), and HMPP (1.9 mol\%). The low level of crosslinking present within this film allows the hydrogel to change its volumetric swelling dependent on the external stimuli (Figure 11a). Holographic gratings were fabricated though the recording of interference pattern between a single flash of incident and reflected coherent $355 \mathrm{~nm} \mathrm{Nd}$ :YAG laser light was directed onto samples in a simple reflection grating set up to initiate free radical polymerization of a secondary polymer in ultrafine fringes (Figure 11b,c). Refractive index of the two distinct polymer layers was achieved by increasing the level of crosslinking in the secondary polymer (HEMA, $13 \mathrm{~mol} \%$, ethylene glycol dimethacrylate (84 mol\%), HMPP (3 mol\%)). The benefit of this "double polymer" system is the lack of requirement for chemical developments post exposure. In comparison to AgNP systems where development and fixation are required the sensors developed within this work only require a simple washing in an ethanol water bath $(1: 1, \mathrm{v} / \mathrm{v})$ step to prepare samples for use. This streamlines the synthesis process, increasing applicability to mass production and widespread use. Sensors demonstrates a clear color shift for the detection of alcohols up to $60 \%$, with a higher sensitivity to isopropanol when compared to other short chain alcohols (Figure 11d). Although this higher level of shifting was observed, the response time went in contrary to this, with shorter alcohol chains showing the faster rate of equilibration, isopropanol (15 $\mathrm{min}$ ) and methanol (7 $\mathrm{min})$, attributed to a faster rate of diffusion within the film. The sensors clear color changes are distinguishable with the naked eye but offer the opportunity for quantitative analysis through a spectrophotometer. Overall, the sensors developed in this work show a strong response to the variation of alcohol concentration through a simple double polymer fabrication.

\subsection{Gas Monitoring}

The monitoring for the presence of potentially harmful gases has applicability to a wide range of industries such as workplaces ${ }^{[95]}$ with even minor exposures showing detrimental effects on health. ${ }^{[96]}$ Rho et al. produced a responsive liquid crystalline holographic meta surface using photonic structure casting to create an optical sensor capable of determining the presence of volatile organic compounds giving a clear image response to the viewer. ${ }^{[97]}$ A meta surface hologram fabricated 
a)

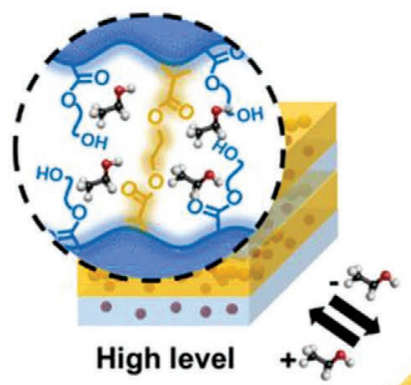

b)
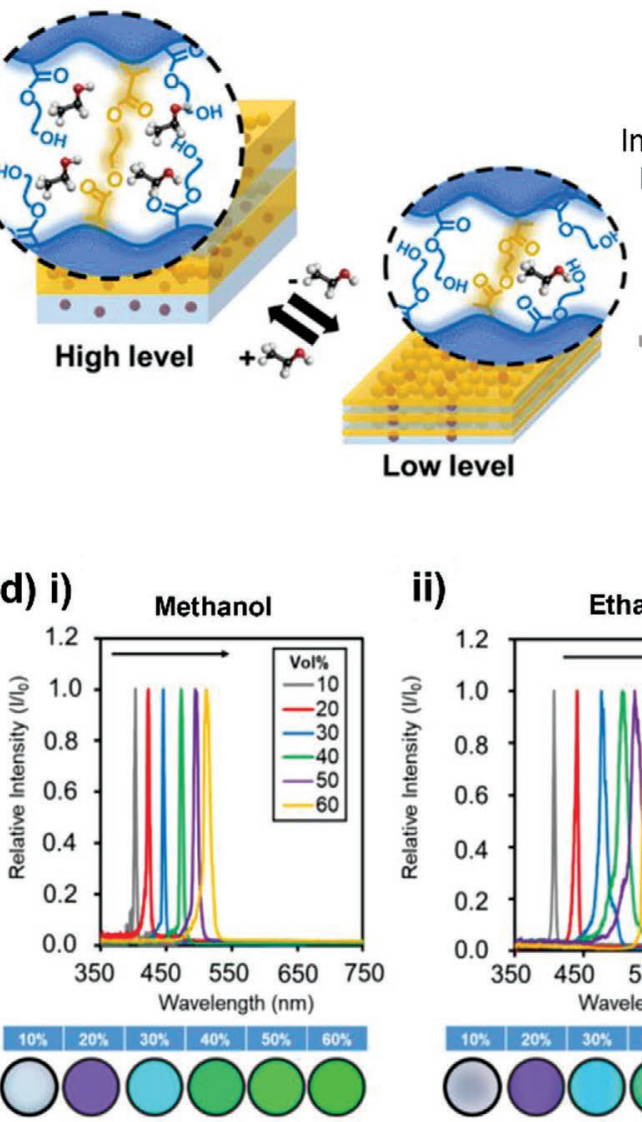

ii)

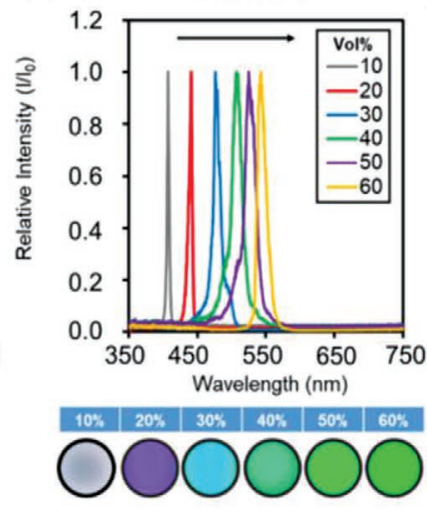

c) Relative

intensity (a.u) Effective Light intensity

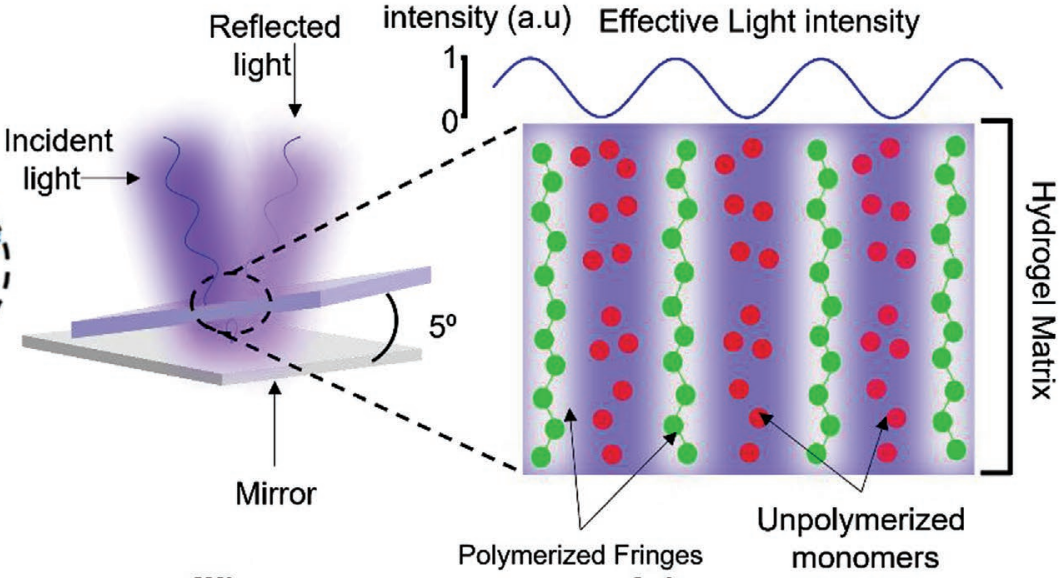

iii)

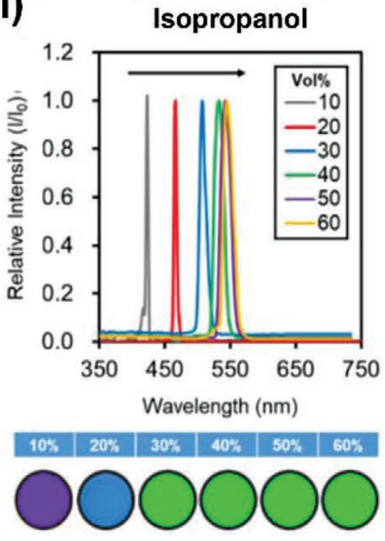

iv)

1-Propanol

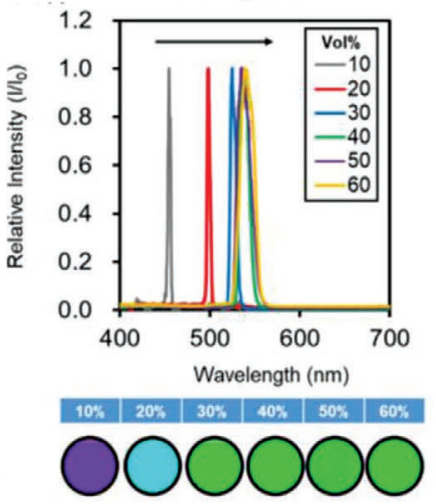

Figure 11. Short chain alcohol sensor fabricated via double photopolymerization of hydrogels. a) Influence of increasing alcohol concentration of $5^{\circ}$; polymer layer swelling; b) Incident and reflected coherent laser light with the hydrogel sample raised to c) Effective laser light intensity passing through the film from constructive and destructive interference and its polymerization effect on the hydrogel film; d) Bragg peak shift and respective color variation observed for short chain alcohols i) methanol, ii) ethanol, ii) isopropanol, and iv) 1-propanol. Reproduced with permission. [73] Copyright 2021, American Chemical Society.

from hydrogenated amorphous silicon was utilized to generate alternative images dependent on the polarization of incident light (Figure 12a,b). The one step fabrication process utilizing spin coating of polyethylene terephthalate on to a range of substrates to cast a photonic mold produced optically active films post polymerization. To alter the polarization of incident light a responsive liquid crystal material 4-cyano-4'-pentylbiphenyl. Light passing through the nematic liquid crystal phase varied the passage of either left or right circularly polarized light, illuminating either "alarm" or "safe" holographic images respectively (Figure 12c). Upon introduction of volatile compounds, the ordered structure is disrupted which reduces the retardation for right circularly polarized light to pass through the device. Sensors identified the presence of many common organic volatiles such as chloroform, acetone, and toluene with response time of $1.3,1.6$, and $5.2 \mathrm{~s}$, respectively. Whilst also maintaining high reversibility between the "alarm" and "safe" messaging, allowing for sensors to be left in situ and report real time variation in air contamination. The devices developed in this research are low cost with simple manufacturing processes, rapid response times, simple image readout, and high reversibility without the requirement for electrical or mechanical components.

\subsection{Glucose Monitoring}

With the pandemic rise in diabetes, glucose has become one of the most monitored metabolites worldwide. ${ }^{[98]}$ Although treatments are improving in dealing with a range of diabetic illnesses, identification of diabetic variation is vital. ${ }^{[9]}$ Current technologies such as, finger prick testing, although accurate have several points for improvement. ${ }^{[100]} \mathrm{A}$ core drawback with these devices is the invasive nature, requiring the breaking of skin and bruising leading to patient discomfort and over time fatigue with the monitoring process. Noninvasive techniques have become a major point of interest for sensing to try and combat monitoring fatigue. ${ }^{[101]}$ Butt et al. developed imprinted hydrogel sensors, capable of rapid continuous and quantitative blood glucose concentrations determination utilizing phenylboronic acid (PBA) functionalized hydrogel films. The 2.5D optical imprinted structure casting method utilized is 


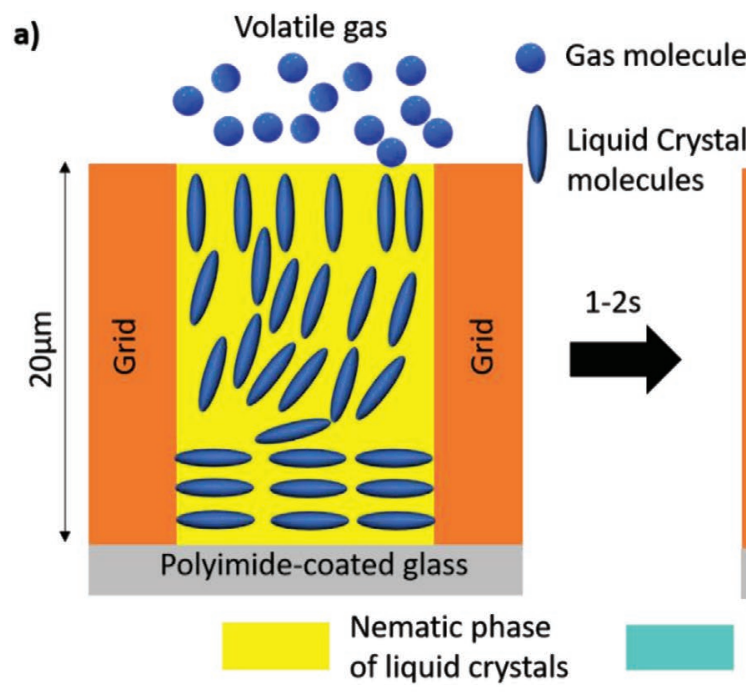

b)

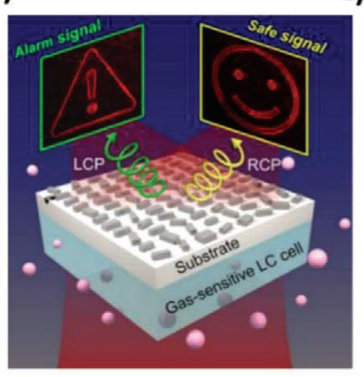

c) i)

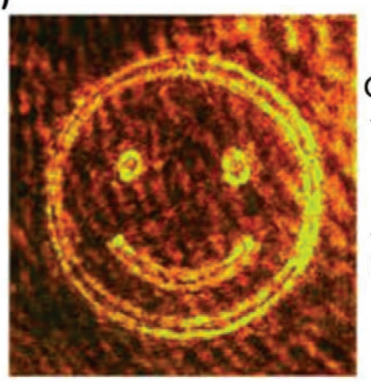

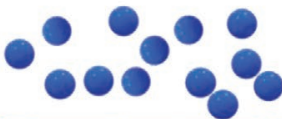

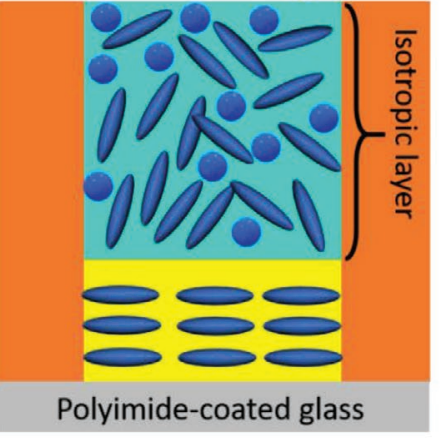

Isotropic phase of liquid crystals

ii)

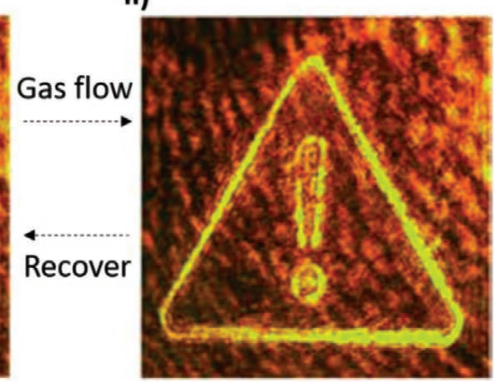

Figure 12. Optical volatile organic gas sensor based on liquid crystal meta surface holograms, a) functional properties of the phase change within the gas sensitive LC cell upon introduction of volatile gas compounds; b) The fundamentals of how light interacts with the sensor illustrating either the 'alarm' or 'safe' signage; c) Experimental result of introduction of gas molecules to sensor surface when illuminated with $600 \mathrm{~nm}$ light. Reproduced with permission. ${ }^{[97]}$ Copyright 2021, American Association for the Advancement of Science.

illustrated in Figure 13a. ${ }^{[54]}$ These devices counter the infrequency in measurements, and give colorimetric responses analyzed quantitatively with a mobile phone camera. This reduces the requirement for sophisticated and expensive equipment to obtain reliable results. The hydrogel matrices employed reversibly change their volume upon on the binding of glucose to the PBA modified hydrogel which has been imprinted with an optically active honeycomb $2.5 \mathrm{D}$ structure by casting a polymer on a PDMS stamp. The fabrication process is simple and low cost, which is readily scalable to a mass production level, due to only requiring minimal processing. The honeycomb structure imprinted on the hydrogel matrix can interact with light to give 1st and 2nd order refractions, the angle of this refraction is indicative of the glucose concentration present in the analyte (Figure 13b). This work was able to demonstrate reliable results within a 15-min analysis window.

Binding of glucose to PBA groups embedded within the matrix leads to an overall expansion of the imprinted structure on the hydrogel surface and therefore the response observed when interacting with light (Figure 13c). Boronic acids are efficient in the reversible binding of cis diol groups which are present on several common sugars such as glucose, fructose, galactose, and mannose. The mechanism of binding is dependent on several factors such as $\mathrm{pH}$ and $\mathrm{pK}_{\mathrm{a}}$ of the boronic acid group. At $\mathrm{pH}>10$ the boronic acid group is anionic and in the tetrahedral conformation where there is a higher efficacy for diol binding. ${ }^{[102]}$ James et al. identified that by positioning a tertiary amine within the boronic acid monomer it is possible to bring down the $\mathrm{pK}_{\mathrm{a}}$ of the group therefore allowing for binding at physiological $\mathrm{pH}$ values. ${ }^{[103]}$

A glucose sensor was fabricated by Jiang et al., using silver halide chemistry and laser interference lithography (LIL). ${ }^{[105]}$ The hydrogel matrix was sensitized to glucose utilizing a 3-acrylamido phenylboronic acid (3-AAPBA). A monomer solution containing AM, PEGDA, 3-AAPBA, and 2-HMP in DI water was pipetted on a silanized glass slide. The hydrogel film was formed by free-radical polymerization under UV exposure for $3 \mathrm{~min} . \mathrm{Ag}^{+}$ions were diffused into the synthesized hydrogel film which was then exposed to a solution containing $\mathrm{LiBr}$ and photosensitive acridine orange dye (later removed from the matrix), which converted $\mathrm{Ag}^{+}$ions to photosensitive $\mathrm{AgBr}$ nanocrystals (NCs) (Figure 14a). Reflection grating was recorded using a continuous wave $(\mathrm{CW})$ laser diode $(\lambda=532 \mathrm{~nm}, 5 \mathrm{~mW}$ ) (Figure 14b). The fabrication process took less than $10 \mathrm{~min}$. The hydrogel was shaped into round flakes of diameter $2 \mathrm{~mm}$. The measurements of hydrogel response to glucose solutions were performed using a spectrophotometer 
a)

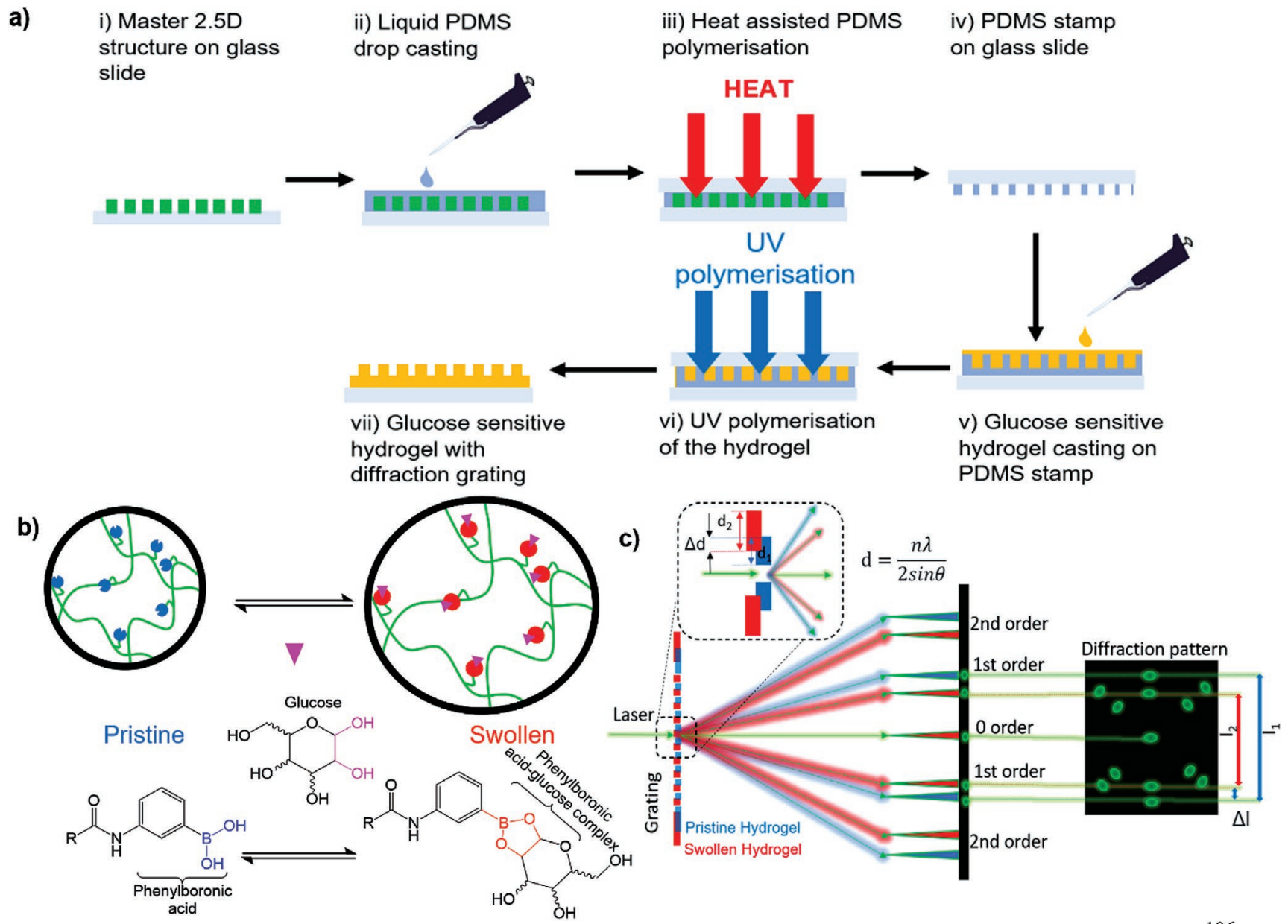

ii) Liquid PDMS

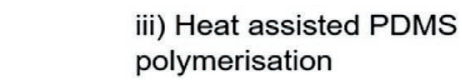

iv) PDMS stamp

polymerisation

on glass slide

Figure 13. Boronic acid-sensitive photonic sensors by molecularly imprinted casting. a) Fabrication process of 2.5D molecularly imprinted polymers via casting of PDMS stamp; b) Equilibrium reaction profile of the boronic acid binding mechanism; c) The principle of the diffraction mechanism observed with 2.5D imprinted sensors when swollen (wet) or pristine (dry); Reproduced under the terms and conditions of the Creative Commons Attribution (CC BY) license. ${ }^{[54]}$ Copyright 2017, published by RSC.

under broadband light. Glucose-boronic acid complexation resulted in increased Donnan osmotic pressure of the system and hydrogel swelling. The response of the sensor to glucose was tested in the applicable physiological range $0-20 \mathrm{mmol} \mathrm{L}^{-1}$. As the glucose concentration increased by 5 and $20 \mathrm{mmol} \mathrm{L}^{-1}$, the hydrogel flake diameter expanded by $1.4 \%$ and $7.7 \%$, respectively, within $40 \mathrm{~min}$ (Figure 14c). The Bragg peak shifted by $5 \mathrm{~nm}$ and $12 \mathrm{~nm}$, respectively, within $1 \mathrm{~h}$ (Figure 14d). Without functionalizing the hydrogel with 3-AAPBA molecules, the flakes showed no significant expansion. The average sensitivity was $0.2 \mathrm{mmol} \mathrm{L}^{-1}$. The response of the hydrogel was found to be reversible. Selectivity studies showed that the hydrogel responded by expansion, due to contact with fructose and lactate solutions as well (Figure 14e). The sensor offers costeffective sensing method for glucose within the applicable clinical range. The fabrication is quick and has the potential to be implemented on mass scale. The hydrogel matrix could be functionalized for detection of other analytes. There are some improvements to be made, such as increasing the diffraction efficiency above $9 \%$ and improving the selectivity for glucose.
Lowe et al. developed a novel methodology for the formulation of holographic gratings via a double polymerisation mechanism. ${ }^{[72]}$ The approach utilizes an initial polymer layer which is lightly crosslinked (P1), into this layer a secondary monomer solution is soaked in which contains a significantly higher crosslinker concentration. The secondary polymer (P2) is polymerized via exposure to reflected laser light where constructive and destructive interference between incident and reflected waves of light generate a holographic grating. ${ }^{[72]}$ The holographic grating is generated due to the difference in refractive index between the two polymer layers within the film. The functionalization group utilized within this work is a boronic acid bearing ligand 3-AAPBA, capable of binding glucose and other carbohydrates via cis diols present on the structure. It is described that the hologram responses were complicated due to the two polymer components displaying initially an expansion followed by a contraction response to the presence of glucose, variation in results observed would be time dependent. The initial expansion effect was attributed to the formation of a negative charge on the boronic acid which in turn led to the rapid penetration of $\mathrm{H}_{2} \mathrm{O}$ and cations into the film to balance 
a)

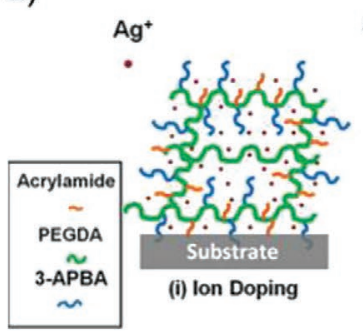

c)

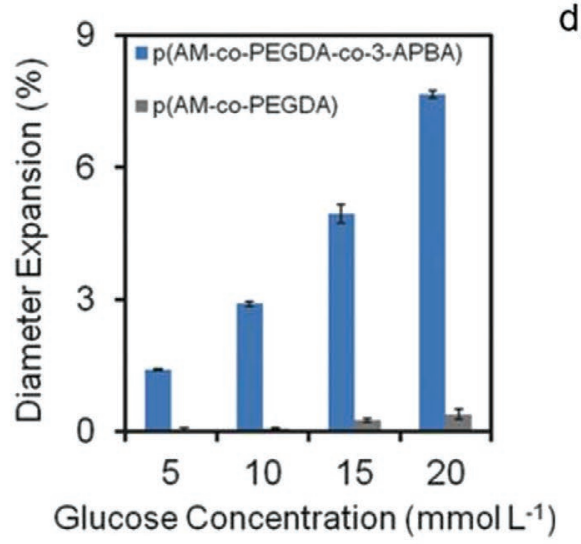

Acridine orangecoated $\mathrm{AgBr}$
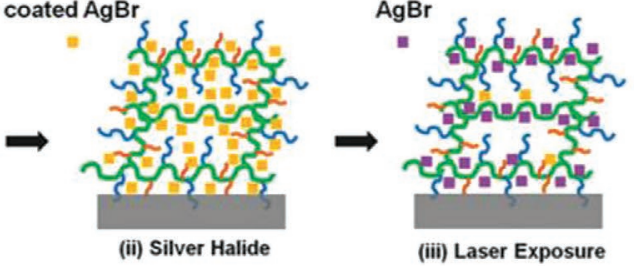

b)

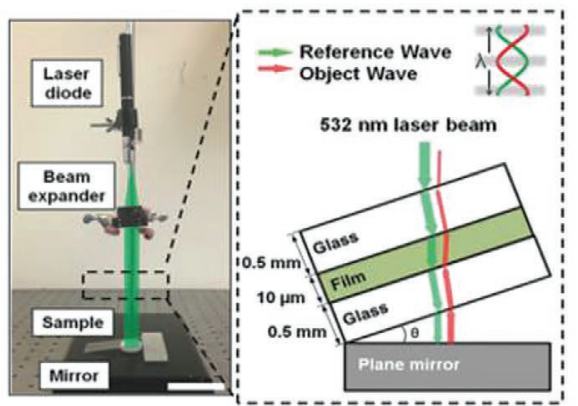

e)

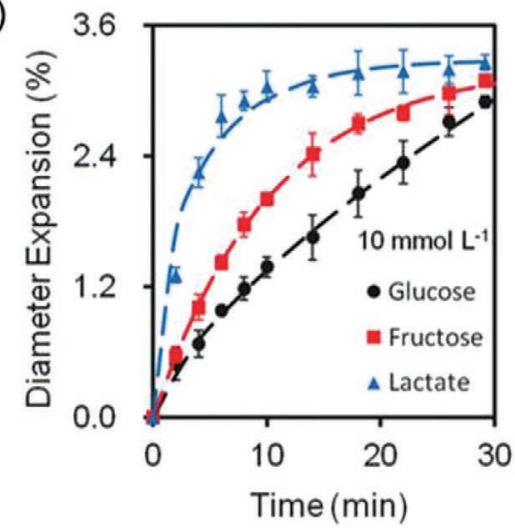

Figure 14. Boronic acid glucose sensor fabricated through silver nanoparticle photolithography. a) Sensor fabrication; b) Recording of the setup for recording of reflection grating (scale bar $=5.0 \mathrm{~cm}$ ); Reproduced with permission from Elsevier; c) Diameter expansion of the 3-AAPBA functionalized and non-functionalized hydrogel versus glucose concentration; d) Bragg peak shift of the 3-AAPBA functionalized hydrogel in time at different glucose concentrations; e) Response of the sensor in time to glucose, fructose and lactate. Reproduced with permission. ${ }^{[105]}$ Copyright 2018, Wiley-VCH.

the anionic charge generated in the 1:1 binding reaction. The contracting response is hypothesized to be due to the sluggish formation of a 1:2 binding complex with glucose until an equilibrium was achieved. To overcome these response variations, Lowe et al. added an equimolar equivalent of glucose to the P1 film formation which was then subsequently removed prior to P2 soaking, this effort to imprint the polymer with glucose was to ensure the spatial positioning of boronic acid groups would more easily facilitate the formation of 1:2 complexes upon binding. The results obtained from this work followed this hypothesis where the equilibration point was achieved more rapidly but the wavelength shift was significantly decreased. Although this methodology showed promise it is thought that at higher concentrations there will be a preference for the 1:1 conformation due to the excess of glucose present within the analyte. The response time for these films varied formulation but at approximately $20 \mathrm{~min}$ an equilibration point was reached. These sensors have been capable of showing a novel double polymer mechanism of forming holographic films is viable, but more work is required to bring their specificity and the equilibration of the response time down to more actionable values.

\subsection{Hormone Sensing}

Mayes et al., demonstrated a novel methodology for detecting testosterone whereby molecularly imprinted films were generated by directly structuring metal free holographic gratings within polymer substrates during synthesis. ${ }^{[106]}$ By recording holograms within a molecularly imprinted (MIP) film selectivity was achieved, this partnered with the visible color response of a holographic grating offers the opportunity for the determination of the presence of analytes. The paper published utilizes complex precursor solutions consisting of imprinting factors (IF), functional monomers, crosslinkers, and photoinitiators to formulate the porous MIP structures, into the polymer based optical gratings are recorded utilizing an Nd:YAG Laser $(\lambda=532 \mathrm{~nm})$. The sensor described within this work demonstrated a selectivity towards testosterone when compared to a competing analyte ( $5 \alpha$-androstane). When comparing these two analytes the chirality and backbone structure is comparable, but due to the absence of the alcohol and ketone group on the $5 \alpha$-androstane the affinity experienced is significantly weakened when compared to testosterone, allowing for the higher selectivity.

\subsection{Cocaine Detection}

Illicit drugs are a significant problem in society due to their production and sale funding criminal groups endeavours. ${ }^{[107]}$ At present law enforcement officers utilize a simple colorimetric dye to identify the presence of cocaine on surfaces, which show low selectivity and can incorrectly identify substances. ${ }^{[108]}$ Conventional nuclear magnetic imaging techniques are 
a) i)<smiles>COC(=O)[C@H]1CCC[C@@H]1COC(=O)c1ccccc1</smiles>

Cocaine

ii)<smiles>CC(C)(C)OC(=O)CCCc1ccc2c(c1)C(=O)OC21c2ccc(O)cc2Oc2cc(O)ccc21</smiles>

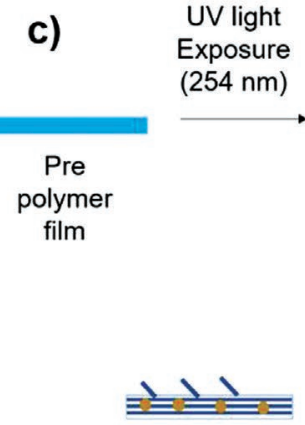

Hydrogel film with holographic grating

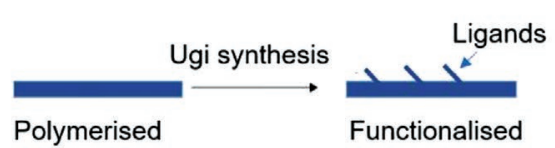
hydrogel film

6 ns pulse from green laser beam $(532 \mathrm{~nm})$

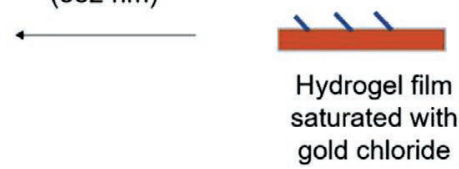

b)

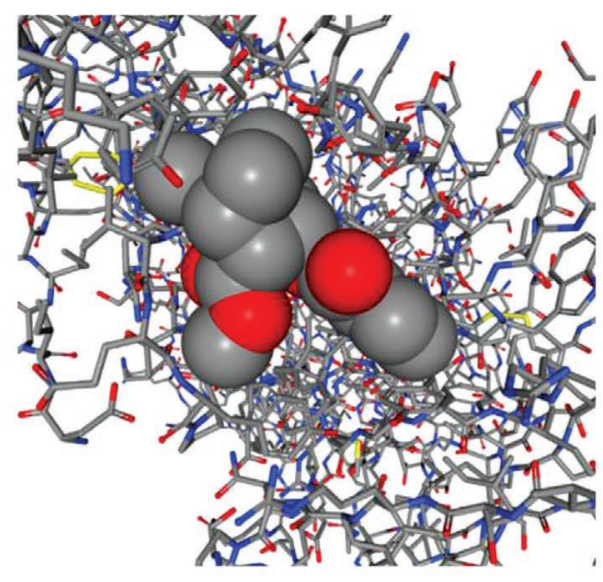

d)

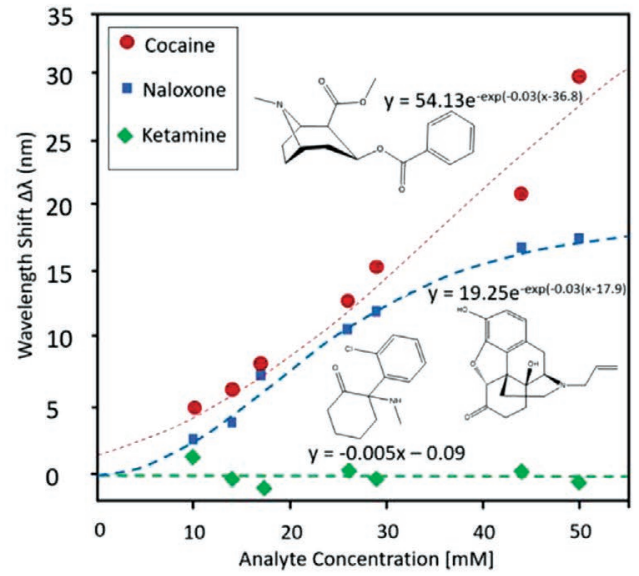

Figure 15. Ugi ligand cocaine sensor fabricated utilizing gold nanoparticle holographic sensors. a-i) Structure of cocaine ii) Structure of the A19C2815 ligand synthesized on the holographic matrix; b) X-ray crystallography of the A19C2815 ligand interacting with cocaine; c) Schematic of sensor fabrication; d) Response of the sensor to cocaine, naloxone and ketamine concentrations within $10-50$ mmol L-1. Reproduced with permission. ${ }^{[68]}$ Copyright 2018, Elsevier B.V.

impractical due to device size, safety hazards, and complexity demonstrating the requirement for selective analytical techniques which can be used in field locations. Due to holographic sensors low cost and high sensitivity chemical sensing platform the sensors have found application in detection of illicit drugs. Oliveira et al. developed a sensor for cocaine detection basing on Ugi synthesis of a ligand to mimic cocaine-protein interactions and Bragg diffraction grating comprising gold nanoparticles. ${ }^{[68]}$ Cocaine (Figure 15a -i) was found to interact with human carboxylesterase-1 (hCE1) and catalytic antibody GNL7A1. Screening of the combinational library for compounds for Ugi synthesis aimed at producing a ligand most likely to mimic the cocaine-protein interactions. The formation of Ugi ligand was assessed indirectly by fluorescence microscopy. Eventually, ligand A19C28I5 (Figure 15a -ii) was chosen for this holographic sensor due to its high binding affinity for cocaine (Figure 15b). To fabricate the sensor, polymer film was synthesized on glass slides from a monomer precursor of HEMA (80 mol\%), EGDMA (5 mol\%), and methacrolein (15 mol\%). Monomer solutions were diluted with 1-propanol $(1: 1 \mathrm{v} / \mathrm{v})$, and free radical initiator (DMPA, $1 \% \mathrm{w} / \mathrm{v}$ ) was added. The films were polymerized for $40 \mathrm{~min}$ with a UV lamp (254 nm wavelength). Ugi ligands were synthesized on the polymer films according to a procedure previously described. Gold holograms were fabricated by exposure to a single $6 \mathrm{~ns}$ pulse of a $532 \mathrm{~nm}$ laser (Figure 15c). A smartphone application was also tested for use in cocaine detection by the color response of the sensor.

The response of the sensor to cocaine was tested in the applicable range of $10-50 \mathrm{mmol} \mathrm{L}^{-1}$, with maximum swelling of $\Delta \lambda=30.5 \mathrm{~nm}$ at a concentration of $50 \mathrm{mmol} \mathrm{L}^{-1}$. The limit of detection was found to be $7.1 \mathrm{mmol} \mathrm{L}^{-1}$. The response time of the sensor was determined as $15 \mathrm{~min}$ and the response was found to be reversible. Smartphone readout was also tested and demonstrated to be successful in detecting cocaine in street seized samples. Specificity studies, comparing response of the sensor to cocaine to that of naloxone and ketamine (Figure 15d), showed that the sensor has some response to naloxone, however, less than that to cocaine. The sensor had specific interaction with cocaine at concentrations above $36 \mathrm{mmol} \mathrm{L}^{-1}$ and showed little to no response to ketamine. The sensor offers advantages of appropriate sensitivity, ability to detect real drug samples, short response time, low cost, and reusability. The smartphone-based readout suggested in the paper offers a simple readout method. Utilization of smartphone cameras as analytical devices removes barriers to accessing quantitative analysis. By utilizing applications or add on components, it can 
be possible to achieve analytical chemistry lab quality results using simple paper-based devices. ${ }^{[25]}$

\subsection{Antibiotic Detection}

Molecular imprinted polymers have been a topic of great interest in recent years with the technology offering new avenues in a variety of optical structures to analyze complex analytes. To achieve this binding an IF with similar chemical properties is cast within a polymer mold capable of binding the target analyte. After polymerization, the IF is removed generating a pocket with higher affinity for the analyte due to the special configuration of the binding site (Figure 16a). The powerful technique can be widely applied in a wide array of uses such as chiral compound separations and sensing. ${ }^{[109,110]}$ MIP technology has resulted in issues with capability when scaled to industrial levels but this has not hindered the science being investigated widely with over 4000 patents filed at the WO mentioning MIPs (April, 2020). This technology has been implemented in a wide array of optical applications to create nanostructures within a responsive medium with higher binding affinities for complex analytes.

Antibiotics revolutionized medical treatment of previously fatal illnesses. Although lifesaving, these compounds often lead to pollution of natural resources such as water supplies, sediment, and long-term deposition within the human body. Long term exposure to these compounds can have serious medical implications. ${ }^{[111]}$ This is overshadowed by the risk of development of antimicrobial resistance, by exposing bacteria to low concentrations of antibiotics it is possible for bacterial colonies to amplify resistant strains. ${ }^{[12]}$ This is an imminent risk to modern medicine, if resistance is obtained to core antibiotic scaffolds treatment of common infections could be obsolete. Qiu et al. describe a methodology whereby optical crystal structures can be utilized to indicate the presence of antimicrobials. ${ }^{[113]}$ By utilizing a optical crystalline colloidal array (CCA) which contained molecularly imprinted cavities sensitive to clindamycin hydrochloride (CLI), a common antibiotic for treatment of minor bacterial infections, the paper described a device with a sensitivity range of $0.01-1 \mathrm{mmol} \mathrm{L}^{-1}$ with a response time $<10$ min. ${ }^{[14]}$ Previously to analyze the concentrations of CLI required chemical processing or separation techniques to accurately determine concentrations ${ }^{[115]}$ since then a number of powerful techniques such as HPLC, ${ }^{[116]}$ and mass spectroscopy ${ }^{[117]}$ have been utilized. Although these techniques provide accurate analysis, they require expensive equipment and a trained individual to carry out the analysis, Qiu et al. aimed to overcome this with a point-of-care analysis device. Readouts from the sensor are obtained with the use of a monochromatic blue/violet light source which illuminates the 2D optical crystal giving rise to a Debye ring (Figure 16b).$^{[118]}$ Analyte absorption modifies the spacings within the nanostructured hydrogel, changing the diameter of the Debye ring, with rapid response times of under $10 \mathrm{~min}$ for up to $1 \mathrm{~mm}$ (Figure 16c). Demonstration of the improved sensitivity was shown by comparing the molecularly imprinted colloidal array (MICA) against a nonmolecularly imprinted colloidal array (NICA). It was observed that there was a spacing change of $41 \mathrm{~nm}$ for the MICA and $1 \mathrm{~nm}$ for the NICA with a CLI concentration change of $1 \mathrm{mmol} \mathrm{L}^{-1}$ (Figure 16d). Selectivity is shown to be high, lincomycin, was tested in parallel with CLI to observe the response. Although

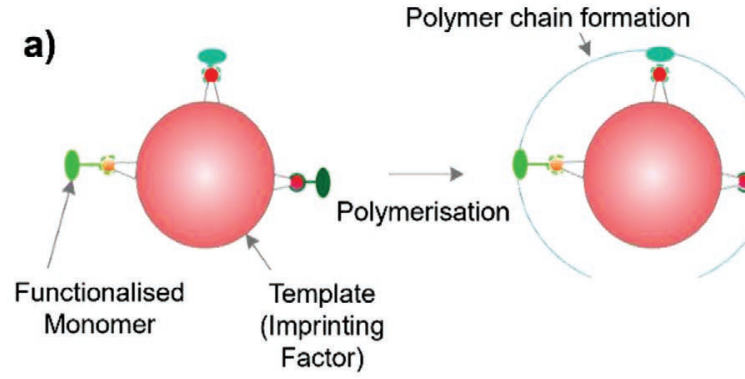

c)

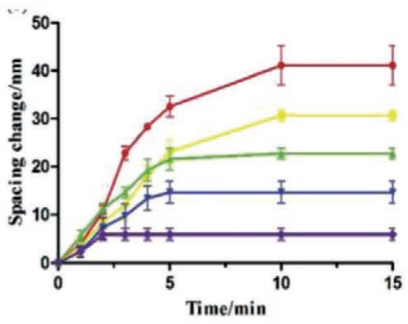

d)

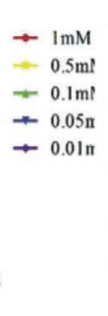

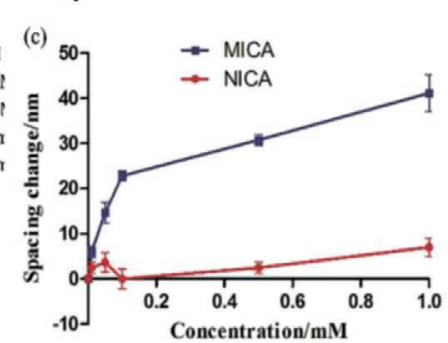

Cavity

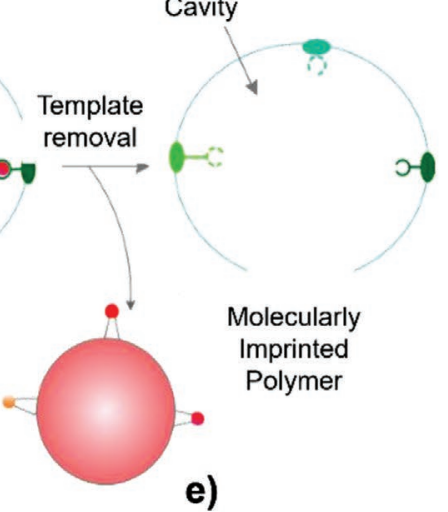

b)

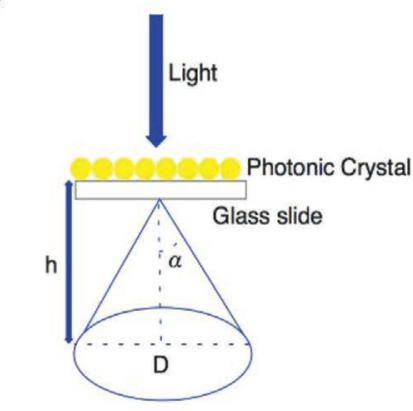

Figure 16. Molecularly imprinted colloidal array antibiotic sensor. a) fabrication of MIP hydrogel films; b) Debye diffraction and a comparison of the change in spacing observed with molecularly imprinted colloidal array (MICA) and non-imprinted colloidal array (NICA); c) Response time of the MICA to CLI at varying concentrations; d) Response of to CLI of both MICA and NICA; e) observable colour change upon exposure to CLI for both the MICA and NICA. Reproduced with permission. ${ }^{113]}$ Copyright 2019, Royal Society of Chemistry.

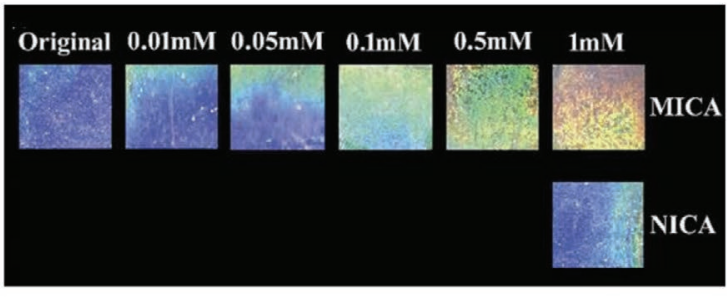


a)

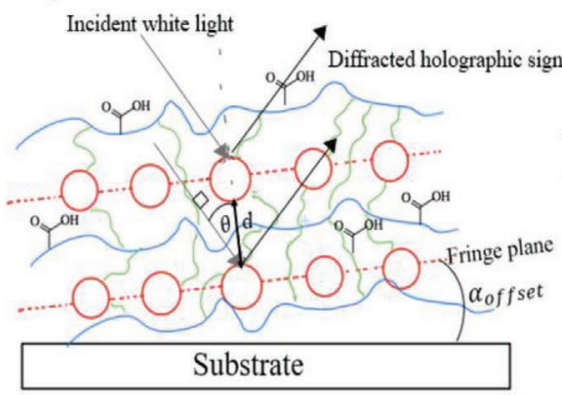

c) i)

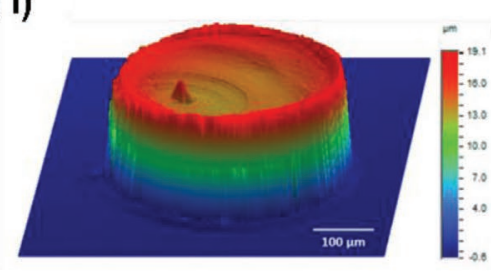

b)

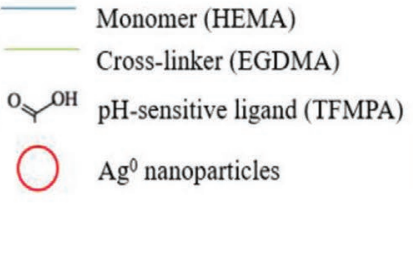

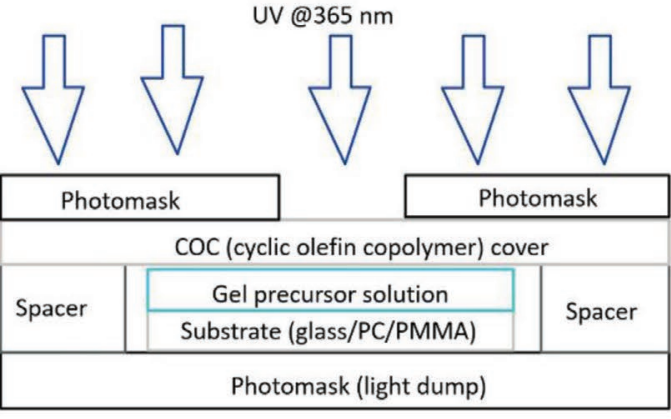

d)

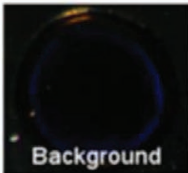

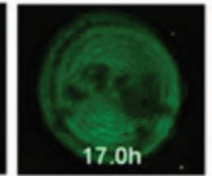
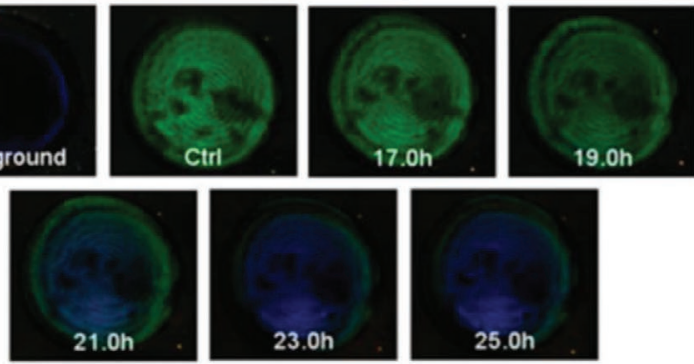
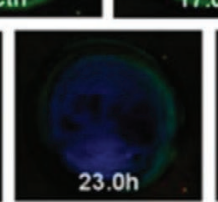

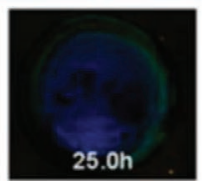

Figure 17. Monitor of bacterial colony growth sensor fabricated through silver nanoparticle photolithography. a) Schematic of the setup for hydrogel fabrication; b) Representation of the sensor function in reflection mode; c-i) Visual representation of 3D elevation of holographic sensor after fabrication, ii) Photograph of holographic sensor taken by CMAS camera whilst immersed in pH 6.0 Mcllvaine buffer; d) Colorimetric response observed upon exposure of holographic gratings to Lactobacillus casei Shirota. Reproduced with permission. ${ }^{120]}$ Copyright 2019 , American Chemical Society.

there was a similar response profile for the presence of lincomycin over concentrations ranging $0.01-1.00 \mathrm{mmol} \mathrm{L} \mathrm{L}^{-1}$ (Figure 15e). The sensor displayed a spacing change of $10 \mathrm{~nm}$ for lincomycin and $40 \mathrm{~nm}$ for CLI at a maximum concentration of $1.0 \mathrm{mmol} \mathrm{L}^{-1}$, demonstrating selectivity for the desired analyte.

The research establishes a methodology whereby the concentration of an antimicrobial contaminate can be identified rapidly at the point of analysis. The sensors binding of analytes is reversible allowing the device to be reusable, overall increasing the availability of the sensor to a wider population due to reduction in costs. Although a clear environmentalist application is intended it is also vital to the medical industry as the effective monitoring of these contaminates in natural resources assists in the mitigation of antimicrobial resistant bacteria occurrence. CLI is chosen as a general antimicrobial within this research but in future research, application of the molecular imprinting fabrication could open an opportunity for optical antimicrobial detection in a range of settings. A further point of research would be the application of these technologies on to urine analysis technologies, by performing these tests on urine monitoring and dosage of antimicrobials to maintain a healthy therapeutic window can be achieved. In tandem this will treat bacterial infections more efficiently, squandering fewer resources and mitigating the risk of emergence antimicrobial resistance. ${ }^{[119]}$

\subsection{Bacterial Growth Monitoring}

A holographic $\mathrm{pH}$ sensor was fabricated by Chan et al. with the purpose of using change in $\mathrm{pH}$ attributed to bacterial metabolism of glucose into lactic acid for monitoring microorganism, Lactobacillus casei Shirota, growth. ${ }^{[120]}$ Gel precursor solution consisting of a monomer: HEMA, cross-linker: EGDMA, solvent: isopropyl alcohol (IPA), pH-sensitive moiety: 2-(trifluoromethyl acrylic acid) (TFMPA), photoinitiator: 2-2-dimethoxy-2-phenylacetophenone (DMPA) and polymerization inhibitor: hydroquinone (HQ) was prepared, introduced between the sample substrate and the COC (cyclic olefin copolymer) cover, and polymerized under UV light through a chrome-glass photomask (Figure 17a). The diffraction gratings were generated via the in situ formation of $\mathrm{Ag}^{0}$ nanoparticles, by diffusing silver perchlorate and lithium bromide into the gel matrix and exposure to a $532 \mathrm{~nm}$ continuous wave SLM laser. The holographic sensor was then enclosed in a microfluidic chip by bonding the substrate with a poly(dimethylsilxone) (PDMS) cover chip with microchannels.

The sensor was tested by spectrometer in reflection mode, ${ }^{[121]}$ the color of reflected light being determined by the alteration of $\mathrm{Ag}^{0} \mathrm{NP}$ fringe spacing at the given $\mathrm{pH}$ (Figure 17b). The gel matrix swells or shrinks depending on the position of equilibrium between protonated and deprotonated form of the $\mathrm{pH}$ sensitive ligand. Here, as $\mathrm{pH}$ increases and leads to a net charge in gel matrix, water and counterions get absorbed in the gel matrix, causing expansion of the gel and increase in the $\mathrm{Ag}^{0}$ nanoparticle interfringe distance, inducing a red shift of the diffracted light. The final fabricated holographic sensors elevation and appearance is illustrated in Figure 17c. Conversely, contraction of the matrix by decreasing $\mathrm{pH}$ result in a blue shift of the diffracted light (Figure 17d). Over a pH range from 3.0 to 6.0 , the peak wavelength of the holographic sensor shifted from 450 to $540 \mathrm{~nm}$. The sensor has accuracy comparable to a standard 
$\mathrm{pH}$ meter and offers a non-invasive method of monitoring of microorganisms in a nanobioreactor, with minimal volume of analytes needed for analysis and simple readout with a camera.

\section{Conclusion}

Holographic sensors cross an intersection of physics, chemistry, and biomedical engineering, as we see research within these respective fields progress the fabrication methods will evolve as they already have improving sensitivity, reliability, and accessibility. Over the past decades, the holographic sensing has been advancing into new fields with sensitivities competing with traditional sensing platforms. Significant progress has been achieved due to the improvement of hydrogel selectivity through advances such as molecular imprinting and improved ligand efficacy, but there is still considerable improvement required to reach a commercialization stage. ${ }^{[122,123]}$ The trend in development of sensors is to improve biocompatibility and remove toxic compounds, such as metal nanoparticles. It has been clearly demonstrated the variety of analytes that can be identified using holographic sensors, from biomarkers including ions, glucose, and temperature to illicit drugs. Quantitative analysis of holographic sensors via smartphones cameras can be utilized to facilitate quantitative analysis in the field. ${ }^{[24]}$ It is clear from current economic trends and the COVID-19 pandemic that personalized medicine and rapid point-of-care devices are going to be a corner stone in future medical treatments. In the past two years we have observed the correlation between the ability for a country to rapidly test its population for COVID-19 and its mortality rate. ${ }^{[125]}$ Holographic sensors can deliver simple colorimetric analysis of biomarkers reliably and could be a great asset in the future detection of illness without the requirement for medical professionals. Fabrication techniques for holographic devices have been summarized, showing simpler low cost, rapid production, and relatively minimal steps required for synthesis, allowing for easier scalability. ${ }^{[126]}$ As presented throughout this review imprinted structures and double polymerized holographic devices are removing the requirement for nanoparticles within holographic devices. Nanoparticles presence in films has hindered holographic sensors use in biomedical applications but the novel techniques described herein abates many health concerns. ${ }^{[127]}$ Response time is a factor that will need to be addressed for the devices to be able to truly function as a biological sensor, at present sensors are dictated by the rate of diffusion throughout the hydrogel to initiate sensing, showing response times too long to be viable, when compared to fluorescent or electrochemical biosensors. ${ }^{[128,129]}$ For holographic devices to achieve their full potential in the application to analyzing biological analytes collaborative works between medical professionals, chemists, and engineers to identify binding targets, design ligands with high specificity to fabricate sensors with ideal characteristics will be required.

\section{Acknowledgements}

A.K.Y. and Y.H. thank the Engineering and Physical Sciences Research Council (EP/T013567/1). N.J. acknowledges the Fundamental Research Funds for the Central Universities (Y)202152).

\section{Conflict of Interest}

The authors declare no conflict of interest.

\section{Keywords}

holography, optical sensors, photolithography, photonics, polymer

Received: June 11, 2021

Revised: July 22, 2021

Published online: August 21, 2021

[1] A. Iadicicco, A. Cutolo, A. Cusano, Adv. Sci. Technol. 2008, 55, 213

[2] H. Tao, M. A. Brenckle, M. Yang, J. Zhang, M. Liu, S. M. Siebert, R. D. Averitt, M. S. Mannoor, M. C. McAlpine, J. A. Rogers, D. L. Kaplan, F. G. Omenetto, Adv. Mater. 2012, 24, 1067.

[3] R. Dersch, M. Steinhart, U. Boudriot, A. Greiner, J. H. Wendorff, Polym. Adv. Technol. 2005, 16, 276.

[4] L. Notte, V. M. N. Passaro, Sens. Actuators, B 2013, 176, 994.

[5] G. Li, C. Liu, X. Zhang, P. Luo, G. Lin, W. Jiang, Food Chem. 2021, $355,129443$.

[6] Q. Zhang, X. Zhang, X. Zhang, L. Jiang, J. Yin, P. Zhang, S. Han, Y. Wang, G. Zheng, Mar. Pollut. Bull. 2019, 144, 20.

[7] S. Han, Q. Zhang, X. Zhang, X. Liu, L. Lu, J. Wei, Y. Li, Y. Wang, G. Zheng, Biosens. Bioelectron. 2019, 143, 111597.

[8] Photonic Sensors Market Research Report - Forecast 2022 | MRFR, https://www.marketresearchfuture.com/reports/photonicsensors-market-2644 (accessed: August 2020).

[9] Photonic Sensors Market Report Analysis, Trends \& Forecast, https://www.alliedmarketresearch.com/photonics-sensor-market (accessed: August 2020).

[10] Clobal Photonics Sensors Market Size, Share | Industry Report, 2022, https://www.grandviewresearch.com/industry-analysis/photonic-sensor-market (accessed: August 2020).

[11] J. W. Galusha, M. R. Jorgensen, M. H. Diamond Bartl, Adv. Mater. 2010, 22, 107.

[12] P. V. Braun, Nature 2011, 472, 423.

[13] R. B. Millington, A. G. Mayes, J. Blyth, C. R. Lowe, Sens. Actuators, B 1996, 33, 55 .

[14] S. Zhang, Y. Chen, Sci. Rep. 2015, 5, 16637.

[15] K. Kertesz, G. Piszter, Z. Balint, L. P. Biro, Sensors 2018, 18, 4282.

[16] A. J. Parnell, A. L. Washington, O. O. Mykhaylyk, C. J. Hill, A. Bianco, S. L. Burg, A. J. C. Dennison, M. Snape, A. J. Cadby, A. Smith, S. Prevost, D. M. Whittaker, R. A. L. Jones, J. P. A. Fairclough, A. R. Parker, Sci. Rep. 2015, 5, 18317.

[17] D. Gabor, Nature 1948, 161, 777.

[18] A. B. Gschwendtner, W. E. Keicher, Lincoln Lab. J. 2000, 12, 383.

[19] L. Cutrona, E. Leith, C. Palermo, L. Porcello, IRE Trans. Inf. Theory 1960, 6, 386.

[20] P. Hariharan, Basics of Holography, Cambridge University Press, Cambridge 2002.

[21] A. K. Yetisen, I. Naydenova, F. da Cruz Vasconcellos, J. Blyth, C. R. Lowe, Chem. Rev. 2014, 114, 10654.

[22] P. Ponikowski, S. D. Anker, K. F. AlHabib, M. R. Cowie, T. L. Force, S. Hu, T. Jaarsma, H. Krum, V. Rastogi, L. E. Rohde, U. C. Samal, H. Shimokawa, B. Budi Siswanto, K. Sliwa, G. Filippatos, ESC Heart Failure 2014, 1, 4.

[23] Y. Khan, A. E. Ostfeld, C. M. Lochner, A. Pierre, A. C. Arias, Adv. Mater. 2016, 28, 4373.

[24] Y. Fuchs, S. Kunath, O. Soppera, K. Haupt, A. G. Mayes, Adv. Funct. Mater. 2014, 24, 688 
[25] X. Xu, A. Akay, H. Wei, S. Wang, B. Pingguan-Murphy, B.-E. Erlandsson, X. Li, W. Lee, J. Hu, L. Wang, F. Xu, Proc. IEEE 2015, 103, 236.

[26] Z. Lei, W. Zhu, X. Zhang, X. Wang, P. Wu, Adv. Funct. Mater. 2021, 31, 2008020.

[27] F. K. Sartain, X. Yang, C. R. Lowe, Anal. Chem. 2006, 78, 5664.

[28] A. K. Yetisen, H. Butt, F. da Cruz Vasconcellos, Y. Montelongo, C. A. B. Davidson, J. Blyth, L. Chan, J. B. Carmody, S. Vignolini, U. Steiner, J. J. Baumberg, T. D. Wilkinson, C. R. Lowe, Adv. Opt. Mater. 2014, 2, 250.

[29] A. Domschke, W. F. March, S. Kabilan, C. Lowe, Diabetes Technol. Ther. 2006, 8, 89 .

[30] A. Domschke, S. Kabilan, R. Anand, M. Caines, D. Fetter, P. Griffith, K. James, N. Karangu, D. Smith, M. Vargas, J. Zeng, A. Hussain, X. Yang, J. Blyth, A. Mueller, P. Herbrechtsmeier C. R. Lowe, Sensors 2004, 3, 1320.

[31] A. G. Mayes, J. Blyth, M. Kyröläinen-Reay, R. B. Millington, C. R. Lowe, Anal. Chem. 1999, 71, 3390.

[32] M. U. Ahmed, I. Saaem, P. C. Wu, A. S. Brown, Crit. Rev. Biotechnol. 2014, 34, 180.

[33] H. K. Brittain, R. Scott, E. Thomas, Clin. Med. 2017, 17, 545

[34] R. M. Turner, B. K. Park, M. Pirmohamed, Wiley Interdiscip. Rev.: Syst. Biol. Med. 2015, 7, 221.

[35] M. Burns, J. Thromb. Thrombolysis 1999, 7, 137.

[36] H. Yan, C.-J. Yang, N. Tang, Y. Zou, S. Chakravarty, A. Roth, R. Chen, IEEE Sens. J. 2017, 17, 5915.

[37] C. D. Chin, V. Linder, S. K. Sia, Lab Chip 2012, 12, 2118.

[38] Z. Tang, N. Kong, X. Zhang, Y. Liu, P. Hu, S. Mou, P. Liljeström, J. Shi, W. Tan, J. S. Kim, Y. Cao, R. Langer, K. W. Leong, O. C. Farokhzad, W. Tao, Nat. Rev. Mater. 2020, 5, 847.

[39] F. Taffoni, D. Formica, P. Saccomandi, G. Di Pino, E. Schena, Sensors 2013, 13, 14105.

[40] J. L. Martínez-Hurtado, C. A. B. Davidson, C. R. Lowe, in SPIE Proc., Advanced Environmental, Chemical, and Biological Sensing Technologies VIII, Vol. 8024, SPIE, Bellingham, WA 2011.

[41] G. J. Worsley, G. A. Tourniaire, K. E. S. Medlock, F. K. Sartain, H. E. Harmer, M. Thatcher, A. M. Horgan, J. Pritchard, J. Diabetes Sci. Technol. 2008, 2, 213.

[42] H. Oikarinen, A. Karttunen, E. Pääkkö, O. Tervonen, Insights Imaging 2013, 4, 729.

[43] B. A. Hargreaves, P. W. Worters, K. B. Pauly, J. M. Pauly, K. M. Koch, G. E. Gold, Am. J. Roentgenol. 2011, 197, 547.

[44] V. R. Varma, A. M. Oommen, S. Varma, R. Casanova, Y. An R. M. Andrews, R. O'Brien, O. Pletnikova, J. C. Troncoso, J. Toledo, R. Baillie, M. Arnold, G. Kastenmueller, K. Nho, P. M. Doraiswamy, A. J. Saykin, R. Kaddurah-Daouk, C. LegidoQuigley, M. Thambisetty, PLoS Med. 2018, 15, e1002482.

[45] M. Anaya, A. Rubino, M. E. Calvo, H. Míguez, J. Mater. Chem. C 2016, 4, 4532.

[46] I. Naydenova, Holographic Sensors, Elsevier, Amsterdam 2020, pp. 165

[47] A. K. Yetisen, H. Butt, L. R. Volpatti, I. Pavlichenko, M. Humar, S. J. J. Kwok, H. Koo, K. S. Kim, I. Naydenova, A. Khademhosseini, S. K. Hahn, S. H. Yun, Biotechnol. Adv. 2016, 34, 250.

[48] J. B. Rosolem, C. F. Barbosa, C. Florídia, E. L. Bezerra, in SPIE Proc., vol. 7503, SPIE, Bellingham, WA 2009

[49] T. Hirschfeld, T. Deaton, F. Milanovich, S. Klainer, Opt. Eng. 1983, 22, 527.

[50] S. Di Carlo, M. Falasconi, Advances in Chemical Sensors, Intech Open, London, UK 2012.

[51] M. Zawadzka, T. Mikulchyk, D. Cody, S. Martin, A. K. Yetisen, J. L. Martinez-Hurtado, H. Butt, E. Mihaylova, H. Awala, S. Mintova, S. H. Yun, I. Naydenova, Photonic Materials for Holographic Sensing BT - Photonic Materials for Sensing, Biosensing and
Display Devices (Eds: M. J. Serpe, Y. Kang, Q. M. Zhang), Springer International Publishing, Cham 2016, pp. 315-359.

[52] M. T. Gale, Microelectron. Eng. 1997, 34, 321.

[53] Y. J. Liu, X. W. Sun, Adv. Optoelectron. 2008, 2008, 684349.

[54] M. Bajgrowicz-Cieslak, Y. Alqurashi, M. I. Elshereif, A. K. Yetisen, M. U. Hassan, H. Butt, RSC Adv. 2017, 7, 53916.

[55] E. Tian, J. Wang, Y. Zheng, Y. Song, L. Jiang, D. Zhu, J. Mater. Chem. 2008, 18, 1116

[56] Y. Nishijima, K. Ueno, S. Juodkazis, V. Mizeikis, H. Misawa, T. Tanimura, K. Maeda, Opt. Express 2007, 15, 12979.

[57] J. Huang, X. Hu, W. Zhang, Y. Zhang, G. Li, Colloid Polym. Sci. 2008, 286, 113.

[58] G. I. N. Waterhouse, M. R. Waterland, Polyhedron 2007, 26, 356.

[59] E. Rezvani, A. Rafferty, C. McGuinness, J. Kennedy, Acta Biomater. 2019, 94, 145.

[60] Y.-J. Lee, P. V. T. Braun, Adv. Mater. 2003, 15, 563.

[61] R. A. Barry, P. Wiltzius, Langmuir 2006, 22, 1369.

[62] J.-P. Couturier, M. Sütterlin, A. Laschewsky, C. Hettrich, E. Wischerhoff, Angew. Chemie Int. Ed. 2015, 54, 6641.

[63] A. Stein, B. E. Wilson, S. G. Rudisill, Chem. Soc. Rev. 2013, 42, 2763.

[64] A. J. Marshall, J. Blyth, C. A. B. Davidson, C. R. Lowe, Anal. Chem. 2003, 75, 4423 .

[65] S. Kabilan, A. J. Marshall, F. K. Sartain, M.-C. Lee, A. Hussain, X. Yang, J. Blyth, N. Karangu, K. James, J. Zeng, D. Smith, A. Domschke, C. R. Lowe, Biosens. Bioelectron. 2005, 20, 1602.

[66] A. J. Marshall, D. S. Young, J. Blyth, S. Kabilan, C. R. Lowe, Anal. Chem. 2004, 76, 1518

[67] A. G. Mayes, J. Blyth, R. B. Millington, C. R. Lowe, Anal. Chem. 2002, 74, 3649 .

[68] N. C. L. Oliveira, G. E.I Khoury, J. M. Versnel, G. K. Moghaddam, L. S. Leite, J. L. Lima-Filho, C. R. Lowe, Sens. Actuators, B 2018, 270, 216.

[69] A. J. Marshall, D. S. Young, S. Kabilan, A. Hussain, J. Blyth, C. R. Lowe, Anal. Chim. Acta 2004, 527, 13.

[70] J. Yang, X. Zhang, C. Liu, Z. Wang, L. Deng, C. Feng, W. Tao, X. Xu, W. Cui, Prog. Mater. Sci. 2021, 118, 100768.

[71] J. Meng, J. Li, J. Liu, X. Zhang, G. Jiang, L. Ma, Z.-Y. Hu, S. Xi, Y. Zhao, M. Yan, P. Wang, X. Liu, Q. Li, J. Z. Liu, T. Wu, L. Mai, ACS Cent. Sci. 2020, 6, 1431.

[72] G. Khalili Moghaddam, H. Margerison, J. Suzuki, J. Blyth, C. R. Lowe, Sens. Actuators, B 2018, 267, 1.

[73] N. Jiang, S. Davies, Y. Jiao, J. Blyth, H. Butt, Y. Montelongo, A. K. Yetisen, ACS Sens. 2021, 6, 915.

[74] A. K. Yetisen, M. M. Qasim, S. Nosheen, T. D. Wilkinson, C. R. Lowe, J. Mater. Chem. C 2014, 2, 3569.

[75] T. Mikulchyk, J. Walshe, D. Cody, S. Martin, I. Naydenova, Sens. Actuators, B 2017, 239, 776.

[76] K. Kawata, M. Osawa, S. Okabe, Environ. Sci. Technol. 2009, 43, 6046.

[77] D. Ballantyne, P. Scheid, Respir. Physiol. 2001, 129, 5.

[78] L. A. Schneider, A. Korber, S. Grabbe, J. Dissemond, Arch. Dermatol. Res. 2007, 298, 413.

[79] R. Fass, R. Hell, R. E. Sampliner, G. Pulliam, E. Graver, V. Hartz, C. Johnson, P. Jaffe, Dig. Dis. Sci. 1999, 44, 2263.

[80] A. K. Yetisen, H. Butt, S.-H. Yun, ACS Sens. 2016, 1, 493.

[81] Sabad-e-Gul, S. Martin, J. Cassidy, I. Naydenova, in Nanoengineering: Fabrication, Properties, Optics, and Devices XIV (Eds: E. M. Campo, E. A. Dobisz, L. A. Eldada), Vol. 10354, SPIE, Bellingham, WA 2017, pp 47-53.

[82] S.-E. Gul, L. O'Neill, J. Cassidy, I. Naydenova, Sensors 2019, 19, 1026.

[83] N. Van Nhien, N. C. Khan, T. Yabutani, N. X. Ninh, A. Kassu, B. T. M. Huong, T. T. Do, J. Motonaka, F. Ota, Biol. Trace Elem. Res. 2006, 111, 1. 
[84] K. Dzieżyc, T. Litwin, A. Sobańska, A. Członkowska, Neurol. Neurochir. Pol. 2014, 48, 214.

[85] Z. Gong, S. Karandikar, X. Zhang, V. Kotipalli, Y. Lvov, L. Que, in Sensors, IEEE, Piscataway, NJ 2010, p. 29.

[86] I. Naydenova, J. Grand, T. Mikulchyk, S. Martin, V. Toal, V. Georgieva, S. Thomas, S. Mintova, Chem. Mater. 2015, 27, 6097.

[87] R. R. Sartor, M. P. Culmo, J. T. DeWolf, J. Bridge Eng. 1999, 4, 157.

[88] L. Molent, B. Aktepe, Fatigue Fract. Eng. Mater. Struct. 2000, 23, 767.

[89] Y. Zhang, N. Anderson, S. Bland, S. Nutt, G. Jursich, S. Joshi, Sens. Actuators, A 2017, 253, 165.

[90] D. Yu, Q. Liu, Y. He, H. Liu, S. Luo, Opt. Laser Technol. 2021, 143, 107374.

[91] C. Grogan, F. R. McGovern, R. Staines, G. Amarandei, I. Naydenova, Sensors 2021, 21, 1673.

[92] H. Zhou, Z. Wang, W. Zhao, X. Tong, X. Jin, X. Zhang, Y. Yu, H. Liu, Y. Ma, S. Li, W. Chen, Chem. Eng. J. 2021, 403, 126307.

[93] B. AlQattan, D. Benton, A. K. Yetisen, H. Butt, ACS Appl. Mater. Interfaces 2019, 11, 29147.

[94] M. Elsherif, R. Moreddu, M. U. Hassan, A. K. Yetisen, H. Butt, Lab Chip 2019, 19, 2060

[95] R. Pitarma, G. Marques, B. R. Ferreira, J. Med. Syst. 2016, 23, 41

[96] R. B. Dick, Neurotoxicol. Teratol. 1988, 10, 39.

[97] I. Kim, W.-S. Kim, K. Kim, M. A. Ansari, M. Q. Mehmood, T. Badloe, Y. Kim, J. Gwak, H. Lee, Y.-K. Kim, J. Rho, Sci. Adv. 2021, 7, eabe9943.

[98] C. Schmid, C. Haug, L. Heinemann, G. Freckmann, Diabetes Technol. Ther. 2013, 15, 889.

[99] J. Ouyang, X. Ji, X. Zhang, C. Feng, Z. Tang, N. Kong, A. Xie, J. Wang, X. Sui, L. Deng, Y. Liu, J. S. Kim, Y. Cao, W. Tao, Proc. Natl. Acad. Sci. U. S. A. 2020, 117, 28667.

[100] W. Villena Gonzales, A. T. Mobashsher, A. Abbosh, Sensors 2019, $19,800$.

[101] H. Xia, H. Tang, B. Zhou, Y. Li, X. Zhang, Z. Shi, L. Deng, R. Song, L. Li, Z. Zhang, J. Zhou, Sens. Actuators, B 2020, 312, 127962.

[102] W. L. A. Brooks, C. C. Deng, B. S. Sumerlin, ACS Omega 2018, 3, 17863.

[103] M. D. Phillips, T. D. James, J. Fluoresc. 2004, 14, 549.

[104] A. K. Yetisen, H. Butt, T. Mikulchyk, R. Ahmed, Y. Montelongo, M. Humar, N. Jiang, S. Martin, I. Naydenova, S. H. Yun, Adv. Opt. Mater. 2016, 4, 1589.

[105] N. Jiang, H. Butt, Y. Montelongo, F. Liu, S. Afewerki, G. Ying, Q. Dai, S. Yun, A. K. Yetisen, Adv. Funct. Mater. 2018, 28, 1702715.

[106] Y. Fuchs, O. Soppera, A. G. Mayes, K. Haupt, Adv. Mater. 2013, 25, 566.
[107] L. Paoli, Global Crime 2004, 6, 19

[108] M. de Jong, A. Florea, J. Eliaerts, F. van Durme, N. Samyn, K. De Wael, Anal. Chem. 2018, 90,6811.

[109] N. M. Maier, W. Lindner, Anal. Bioanal. Chem. 2007, 389, 377.

[110] W. J. Cheong, F. Ali, J. H. Choi, J. O. Lee, K. Yune Sung, Talanta 2013, 106, 45.

[111] Y. Zhao, Y. Zhou, Q. Zhu, B. Xia, W. Ma, X. Xiao, H. Shi, Y. Zhang, Environ. Int. 2019, 123, 70

[112] S. A. Kraemer, A. Ramachandran, G. G. Perron, Microorganisms 2019, 7, 180

[113] Y. Wang, J. Fan, Z. Meng, M. Xue, L. Qiu, Anal. Methods 2019, 11, 2875

[114] W. Lu, F. Xue, S.-Y. Huang, Z. Meng, M. Xue, Chinese J. Anal. Chem. 2013, 40, 1561.

[115] F. A. El-Yazbi, S. M. Blaih, Analyst 1993, 118, 577.

[116] G. C. Batzias, G. A. Delis, M. Koutsoviti-Papadopoulou, J. Pharm. Biomed. Anal. 2004, 35, 545.

[117] L. L. Yu, C. K. Chao, W. J. Liao, T. Y. Twu, C. M. Liu, T. H. Yang, E. T. Lin, J. Chromatogr. B: Biomed. Sci. Appl. 1999, 724, 287.

[118] N. L. Smith, A. Coukouma, S. Dubnik, S. A. Asher, Phys. Chem. Chem. Phys. 2017, 19, 31813.

[119] J. A. Roberts, R. Norris, D. L. Paterson, J. H. Martin, Br. J. Clin. Pharmacol. 2012, 73, 27

[120] L. C. Z. Chan, G. Khalili Moghaddam, Z. Wang, C. R. Lowe, ACS Sens. 2019, 4, 456

[121] Y. N. Denisyuk, Proc. SPIE 1962, 144, 1275.

[122] Y. Cao, T. Feng, J. Xu, C. Xue, Biosens. Bioelectron. 2019, 141, 111447.

[123] J. Tavakoli, Y. Tang, Polymers 2017, 9, 364.

[124] G. Khalili Moghaddam, C. R. Lowe, PLoS One 2017, 12, e0187467.

[125] P. Pokhrel, C. Hu, H. Mao, ACS Sens. 2020, 5, 2283.

[126] M. S. Khan, M. Rahlves, R. Lachmayer, B. Roth, in 2019 Conf. on Lasers and Electro-Optics Europe Q European Quantum Electronics Conf. (CLEO/Europe-EQEC), IEEE, Piscataway, NJ 2019, p. 1.

[127] P. H. M. Hoet, I. Brüske-Hohlfeld, O. V. Salata, J. Nanobiotechnol. 2004, 2, 12.

[128] H. Teymourian, A. Barfidokht, J. Wang, Chem. Soc. Rev. 2020, 49, 7671.

[129] Y. J. Heo, H. Shibata, T. Okitsu, T. Kawanishi, S. Takeuchi, Proc. Natl. Acad. Sci. U. S. A. 2011, 108, 13399.

[130] D. G. Stavenga, B. D. Wilts, H. L. Leertouwer, T. Hariyama, Phil. Trans. R. Soc. B 2011, 366, 20100197.

[131] A. Didari, M. P. Mengüç, Sci. Rep. 2018, 8, 16891. 


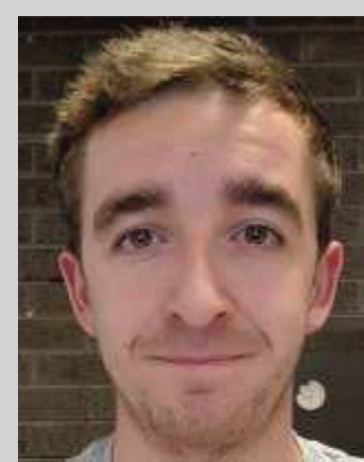

Sam Davies is a Ph.D. student in the Department of Chemical Engineering at Imperial College London. He earned his Bachelor's degree in Chemistry at the University of Liverpool in 2017. He obtained his Master's in Advanced Chemical Sciences at the University of Liverpool in 2019. His current research is focused on the development of holographic devices and nanoparticle free colorimetric devices in the field of biosensing.

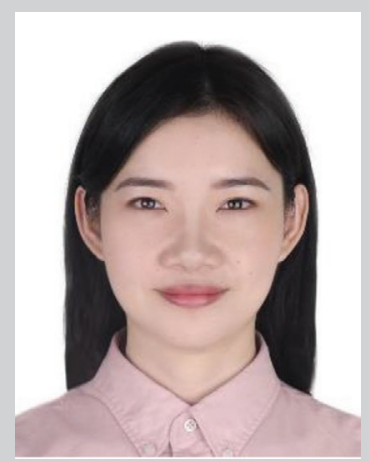

Yubing $\mathrm{Hu}$ is a research associate and assistant supervisor in the Department of Chemical Engineering at Imperial College London. Dr. Hu received a Bachelor's degree from Zhejiang University in 2016 and earned a Ph.D. degree from the Hong Kong University of Science and Technology in 2020. Her Ph.D. study focused on the development of fluorescent polymer materials for advanced sensing and imaging applications. Her current research aims to develop a variety of optical biosensors for wearable diagnostic devices. Her research works have been published in Advanced Materials, Advanced Functional Materials, and CCS Chemistry etc.

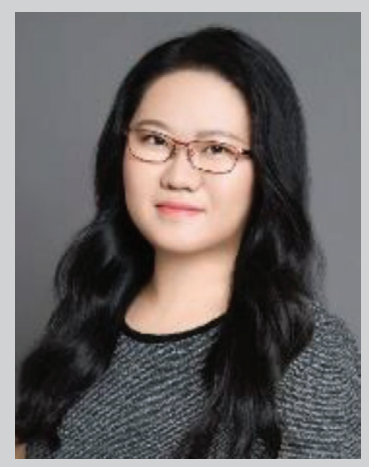

Nan Jiang earned her Ph.D. degree from Wuhan University of Technology. After her Ph.D. study, she worked as a postdoctoral fellow and a research associate in Harvard University and Imperial College London. She is currently working as a faculty member at Sichuan University. Her research is aimed at optical biosensors and microfluidic devices. She has 27 peer-reviewed papers as first author (co-first author) and corresponding author. Some important works have been published on leading journals such as Advanced Materials, Advanced Functional Materials, Energy and Environmental Science, and ACS Nano. Some works have been selected as "Cover paper."

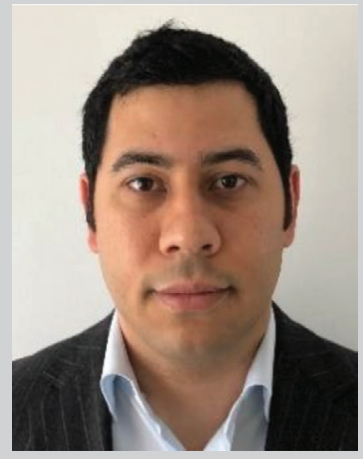

Ali K. Yetisen is a senior lecturer and associate professor in the Department of Chemical Engineering at Imperial College London. He was previously a Tosteson fellow at Harvard University. He holds a Ph.D. degree in Chemical Engineering and Biotechnology from the University of Cambridge. He has been awarded several international prizes including IChemE Nicklin Medal, Birmingham Fellowship, MGH ECOR Award, Humboldt Research Fellowship Award, Carl Friedrich von Siemens Fellowship Award, and a Fellowship of the Royal Society of Chemistry. 